TRANSACTIONS OF THE

AMERICAN MATHEMATICAL SOCIETY

Volume 362, Number 5, May 2010, Pages 2685-2721

S 0002-9947(09)05041-7

Article electronically published on December 15, 2009

\title{
THE SPECTRAL SEQUENCE OF AN EQUIVARIANT CHAIN COMPLEX AND HOMOLOGY WITH LOCAL COEFFICIENTS
}

\author{
STEFAN PAPADIMA AND ALEXANDER I. SUCIU
}

\begin{abstract}
We study the spectral sequence associated to the filtration by powers of the augmentation ideal on the (twisted) equivariant chain complex of the universal cover of a connected CW-complex $X$. In the process, we identify the $d^{1}$ differential in terms of the coalgebra structure of $H_{*}(X, \mathbb{k})$ and the $\mathbb{k} \pi_{1}(X)$-module structure on the twisting coefficients. In particular, this recovers in dual form a result of Reznikov on the mod $p$ cohomology of cyclic $p$-covers of aspherical complexes. This approach provides information on the homology of all Galois covers of $X$. It also yields computable upper bounds on the ranks of the cohomology groups of $X$, with coefficients in a prime-power order, rank one local system. When $X$ admits a minimal cell decomposition, we relate the linearization of the equivariant cochain complex of the universal abelian cover to the Aomoto complex, arising from the cup-product structure of $H^{*}(X, \mathbb{k})$, thereby generalizing a result of Cohen and Orlik.
\end{abstract}

\section{Contents}

1. Introduction

2. The equivariant chain complex of a CW-complex

3. The equivariant spectral sequence

4. Identifying the $d^{1}$ differential

5. Convergence issues

6. Base change and filtrations on homology

7. The homological Reznikov spectral sequence

8. Completion and homology of abelian covers

9. Monodromy action and the Aomoto complex

10. Bounds on twisted cohomology ranks: I

11. Bounds on twisted cohomology ranks: II

12. Galois covers, minimality, and linearization

Acknowledgments

Received by the editors September 29, 2008.

2010 Mathematics Subject Classification. Primary 55N25, 55T99; Secondary 20J05, 57M05.

Key words and phrases. Equivariant chain complex, $I$-adic filtration, spectral sequence, twisted homology, minimal cell complex, Aomoto complex, Betti numbers.

The first author was partially supported by the CEEX Programme of the Romanian Ministry of Education and Research, contract 2-CEx 06-11-20/2006.

The second author was partially supported by NSF grant DMS-0311142.

(C)2009 American Mathematical Society Reverts to public domain 28 years from publication 


\section{INTRODUCTION}

1.1. The equivariant chain complex. In his pioneering work from the late 1940s, J.H.C. Whitehead established the category of CW-complexes as the natural framework for much of homotopy theory. In [34], he highlighted the key role played by the cellular chain complex of the universal cover, $\widetilde{X}$, of a connected CW-complex $X$. Among other things, Whitehead showed that a map $f: X \rightarrow Y$ is a homotopy equivalence if and only if the induced map between equivariant chain complexes, $\tilde{f}_{*}: C_{\bullet}(\tilde{X}, \mathbb{Z}) \rightarrow C_{\bullet}(\tilde{Y}, \mathbb{Z})$, is an equivariant chain-homotopy equivalence. For 2dimensional complexes, the fundamental group, together with the equivariant chainhomotopy type of $C \bullet(\widetilde{X}, \mathbb{Z})$, constitute a complete set of homotopy type invariants: if $X$ and $Y$ are two such spaces, with $\pi_{1}(X) \cong \pi_{1}(Y)$ and $C_{\bullet}(\widetilde{X}, \mathbb{Z}) \simeq C_{\bullet}(\widetilde{Y}, \mathbb{Z})$, then $X \simeq Y$.

As noted by S. Eilenberg [10, the equivariant chain complex is tightly connected to homology with twisted coefficients: given a linear representation, $\rho: \pi_{1}(X) \rightarrow$ $\operatorname{GL}(n, \mathbb{k})$, the homology of $X$ with coefficients in the local system ${ }_{\rho} \mathbb{k}^{n}$ coincides with $H_{*}\left(\rho \mathbb{k}^{n} \otimes_{\mathbb{k} \pi_{1}(X)} C_{\bullet}(\tilde{X}, \mathbb{k})\right)$.

In this paper, we revisit these classical topics, drawing much of the motivation from recent work on the topology of complements of hyperplane arrangements and the study of cohomology jumping loci. One of our main goals is to give tight upper bounds for the twisted Betti ranks, computable in terms of much simpler data, involving only ordinary cohomology. The basic tool for our approach is a spectral sequence, which we now proceed to describe.

1.2. The equivariant spectral sequence. Let $\mathbb{k} \pi$ be the group ring of a group $\pi$, over a coefficient ring $\mathbb{k}$, and let $M$ be a right $\mathbb{k} \pi$-module. The successive powers of the augmentation ideal, $I=I_{\mathbb{k}}(\pi)$, determine a filtration on $M$; the associated graded object, $\operatorname{gr}(M)=\bigoplus_{n \geq 0} M I^{n} / M I^{n+1}$, is a module over the ring $\operatorname{gr}(\mathbb{k} \pi)$.

Now let $X$ be a connected CW-complex, with fundamental group $\pi=\pi_{1}(X)$, and let $C_{\bullet}(X, M)=M \otimes_{\mathbb{k} \pi} C \bullet(\widetilde{X}, \mathbb{k})$ be the equivariant chain complex of $X$ with coefficients in $M$, discussed in $₫ 2$, The $I$-adic filtration on $C_{\bullet}(X, M)$ is compatible with the boundary maps, and thus gives rise to a spectral sequence, $E^{\bullet}(X, M)$, as explained in 93 Under some mild conditions, we identify in $\$ 4$ the differential $d^{1}: E^{1} \rightarrow E^{1}$, solely in terms of the coalgebra structure of $H_{*}(X, \mathbb{k})$ and the $\operatorname{gr}(\mathbb{k} \pi)$ module structure on $\operatorname{gr}(M)$.

Theorem A. There is a second-quadrant spectral sequence, $\left\{E^{r}(X, M), d^{r}\right\}_{r \geq 1}$, with $E_{-p, p+q}^{1}(X, M)=H_{q}\left(X, \operatorname{gr}^{p}(M)\right)$. If $\mathbb{k}$ is a field, or $\mathbb{k}=\mathbb{Z}$ and $H_{*}(X, \mathbb{Z})$ is torsion-free, then $E_{-p, p+q}^{1}(X, M)=\operatorname{gr}^{p}(M) \otimes_{\mathbb{k}} H_{q}(X, \mathbb{k})$, and the $d^{1}$ differential decomposes as

$$
\begin{aligned}
& \operatorname{gr}^{p}(M) \otimes_{\mathbb{k}} H_{q} \stackrel{\operatorname{id} \otimes \nabla_{X}}{\longrightarrow} \operatorname{gr}^{p}(M) \otimes_{\mathbb{k}}\left(H_{1} \otimes_{\mathbb{k}} H_{q-1}\right) \\
& \downarrow \cong \\
& \left(\operatorname{gr}^{p}(M) \otimes_{\mathbb{k}} \operatorname{gr}^{1}(\mathbb{k} \pi)\right) \otimes_{\mathbb{k}} H_{q-1} \stackrel{\operatorname{gr}\left(\mu_{M}\right) \otimes \mathrm{id}}{\longrightarrow} \operatorname{gr}^{p+1}(M) \otimes_{\mathbb{k}} H_{q-1},
\end{aligned}
$$

where $\nabla_{X}$ is the comultiplication map on $H_{*}=H_{*}(X, \mathbb{k})$ and $\mu_{M}: M \otimes_{\mathbb{k}} \mathbb{k} \pi \rightarrow M$ is the multiplication map.

Full details are given in Theorem 4.1. In the case when $X$ is of finite type, $d^{1}$ is determined by the cup-product structure in $H^{*}(X, \mathbb{k})$ and the map $\operatorname{gr}\left(\mu_{M}\right)$. 
The idea to use powers of augmentation ideals to define a second quadrant spectral sequence in terms of group presentations goes back to J. Stallings 32. For more on the Stallings spectral sequence, see [14, 33].

In $\$ 5$, we study the convergence properties of the spectral sequence from Theorem A. Under fairly general assumptions, $E^{\bullet}(X, M)$ has an $E^{\infty}$ term. Yet, as we show in Example 5.3, there are finite $\mathrm{CW}$-complexes $X$ for which $E^{\bullet}(X, \mathbb{k} \pi)$ does not converge.

1.3. Base change. To obtain more structure in the spectral sequence, we restrict ourselves in 86 to a special situation. Suppose $\nu: \pi \rightarrow G$ is an epimorphism onto a group $G$; then the group ring $\mathbb{k} G$ becomes a right $\mathbb{k} \pi$-module, via extension of scalars. The resulting spectral sequence, $E^{\bullet}\left(X, \mathbb{k} G_{\nu}\right)$, is a spectral sequence in the category of left $\operatorname{gr}_{J}(\mathbb{k} G)$-modules, where $J$ is the augmentation ideal of $\mathbb{k} G$. In Proposition 6.3. we describe the differential $d_{G}^{1}$ solely in terms of the induced homomorphism, $\nu_{*}: H_{1}(X, \mathbb{k}) \rightarrow H_{1}(G, \mathbb{k})$, and the comultiplication map, $\nabla_{X}$.

Note that $H_{*}\left(X, \mathbb{k} G_{\nu}\right)=H_{*}(Y, \mathbb{k})$, where $Y \rightarrow X$ is the Galois $G$-cover defined by $\nu$. The homology groups of $Y$ support two natural filtrations: $F^{\bullet}$, coming from the spectral sequence, and $J^{\bullet}$, by the powers of the augmentation ideal. We then have an inclusion, $J^{k} \cdot H_{*}(Y, \mathbb{k}) \subseteq F^{k} H_{*}(Y, \mathbb{k})$. Equality holds for $G=\mathbb{Z}$, as we show in Lemma 8.4, but in general the two filtrations do not agree, even when $G=\mathbb{Z}^{2}$.

In $\$ 8$ we study the more general situation when $\nu: \pi \rightarrow G$ is an epimorphism to an abelian group, making use of the general machinery developed by J.P. Serre in [30]. Assuming $X$ is of finite type and $\mathbb{k}$ is a field, the spectral sequence $E^{\bullet}\left(X, \mathbb{k} G_{\nu}\right)$ converges and computes the $J$-adic completion of $H_{*}\left(X, \mathbb{k} G_{\nu}\right)$. Moreover, if $X$ is a finite CW-complex, the spectral sequence collapses in finitely many steps.

In the case when $X$ is a $K(\pi, 1)$ space, $G=\mathbb{Z}_{p}$, and $\mathbb{k}=\mathbb{F}_{p}$, discussed separately in 97 the spectral sequence $E^{\bullet}\left(X, \mathbb{k} G_{\nu}\right)$ is the homological version of a spectral sequence first considered by A. Reznikov in [27].

1.4. Monodromy action. In $₫ 9$, we focus exclusively on infinite cyclic covers, analyzing the homology groups $H_{q}\left(X, \mathbb{k} \mathbb{Z}_{\nu}\right)$, viewed as modules over the Laurent polynomial ring, $\mathbb{k} \mathbb{Z}=\mathbb{k}\left[t^{ \pm 1}\right]$. We assume $\mathbb{k}$ is a field, so that $\mathbb{k}\left[t^{ \pm 1}\right]$ is a PID. Given an element $a \in H^{1}(X, \mathbb{k})$ with $a^{2}=0$, left-multiplication by $a$ turns the cohomology ring of $X$ into a cochain complex,

$$
\left(H^{*}(X, \mathbb{k}), \cdot a\right): H^{0}(X, \mathbb{k}) \stackrel{a}{\rightarrow} H^{1}(X, \mathbb{k}) \stackrel{a}{\rightarrow} H^{2}(X, \mathbb{k}) \stackrel{a}{\rightarrow} \cdots .
$$

Theorem B. Let $X$ be a connected, finite-type $C W$-complex, $\nu: \pi_{1}(X) \rightarrow \mathbb{Z}$ an epimorphism, and $\nu_{\mathbb{k}} \in H^{1}(X, \mathbb{k})$ the corresponding cohomology class. Then, for all $q \geq 0$ :

(1) The $\operatorname{gr}_{J}(\mathbb{k} \mathbb{Z})$-module structure on $E^{\infty}\left(X, \mathbb{k}_{\nu}\right)$ determines $P_{0}^{q}$ and $P_{t-1}^{q}$, the free and $(t-1)$-primary parts of $H_{q}\left(X, \mathbb{k}_{\nu}\right)$, viewed as $\mathbb{k}\left[t^{ \pm 1}\right]$-modules.

(2) The monodromy action of $\mathbb{Z}$ on $P_{0}^{j} \oplus P_{t-1}^{j}$ is trivial for all $j \leq q$ if and only if $H^{j}\left(H^{*}(X, \mathbb{k}), \cdot \nu_{\mathbb{k}}\right)=0$ for all $j \leq q$.

For a more detailed statement, see Propositions 9.1 and 9.4. Part (2) has an analog for $\nu: \pi_{1}(X) \rightarrow \mathbb{Z}_{p}$ and $\mathbb{k}=\mathbb{F}_{p}(p \neq 2)$; see Proposition 7.5 .

Particularly interesting is the case of a smooth manifold $X$ fibering over the circle, with $\nu: \pi \rightarrow \mathbb{Z}$ the homomorphism induced on $\pi_{1}$ by the projection map, $p: X \rightarrow S^{1}$. The homology of the resulting infinite cyclic cover was studied by 
J. Milnor in 21. This led to another spectral sequence, introduced by M. Farber in [11, and further developed by S.P. Novikov in [22]. The Farber-Novikov spectral sequence has an $\left(E_{1}, d_{1}\right)$-page dual to our $\left(E^{1}\left(X, \mathbb{k} \mathbb{Z}_{\nu}\right), d_{\nu}^{1}\right)$-page, and higher differentials given by certain Massey products; see [12. Their spectral sequence, though, converges to the free part of $H_{*}\left(X, \mathbb{k} \mathbb{Z}_{\nu}\right)$, and thus misses the information on the $(t-1)$-primary part captured by the equivariant spectral sequence. The spectral sequence $E^{\bullet}\left(X, \mathbb{k} \mathbb{Z}_{\nu}\right)$ was also investigated by $\mathrm{G}$. Denham in $[\underline{6}$, in the special case when $X$ is the complement of a complexified arrangement of real hyperplanes through the origin of $\mathbb{R}^{\ell}$ and when $p$ is the Milnor fibration.

Theorem $\mathrm{B}$ has already found several applications in the literature. In [24], we analyze the monodromy action on the homology of Galois $\mathbb{Z}$-covers, for toric complexes associated to finite simplicial complexes. Using part (2) of the theorem, we obtain a combinatorial criterion for the full triviality of this action, up to a given degree. In 25, Theorem B(2) yields a new formality criterion and a purely topological proof of a basic result on the monodromy action, for the homology of Milnor fibers of plane curve singularities.

1.5. Bounds on twisted Betti ranks. Computing cohomology groups with coefficients in a rank 1 local system can be an arduous task. It is thus desirable to have efficient, readily computable bounds for the ranks of these groups. We supply such bounds in $\S \S 1011$.

Given a non-zero complex number $\zeta \in \mathbb{C}^{\times}$and a homomorphism $\nu: \pi \rightarrow \mathbb{Z}$, define a representation $\rho: \pi \rightarrow \mathbb{C}^{\times}$by $\rho(g)=\zeta^{\nu(g)}$. If $\zeta$ is a $d$-th root of unity, such a homomorphism $\rho$ is called a rational character (of order $d$ ).

Theorem C. Let $X$ be a connected, finite-type $C W$-complex, and let $\rho: \pi_{1}(X) \rightarrow$ $\mathbb{C}^{\times}$be a rational character of prime-power order $d=p^{r}$. Then, for all $q \geq 0$,

$$
\operatorname{dim}_{\mathbb{C}} H^{q}\left(X,{ }_{\rho} \mathbb{C}\right) \leq \operatorname{dim}_{\mathbb{F}_{p}} H^{q}\left(X, \mathbb{F}_{p}\right) .
$$

If, moreover, $H_{*}(X, \mathbb{Z})$ is torsion-free,

$$
\operatorname{dim}_{\mathbb{C}} H^{q}\left(X, \rho_{\mathbb{C}}\right) \leq \operatorname{dim}_{\mathbb{F}_{p}} H^{q}\left(H^{*}\left(X, \mathbb{F}_{p}\right), \nu_{\mathbb{F}_{p}}\right) .
$$

The proof is given in Theorems 10.3 and 11.3. Neither of these two inequalities can be sharpened further: we give examples showing that both the prime-power order hypothesis on $d$ and the torsion-free hypothesis on $H_{*}(X, \mathbb{Z})$ are really necessary.

The second inequality above generalizes a result of D. Cohen and P. Orlik (4, Theorem 1.3]), valid only when $X$ is the complement of a complex hyperplane arrangement and $r=1$. This inequality is used in a crucial way in [19. It yields a purely combinatorial description of the monodromy action on the degree 1 rational homology of the Milnor fiber for arbitrary subarrangements of classical Coxeter arrangements.

1.6. Minimality and linearization. Using the equivariant spectral sequence of a Galois cover, we give in $\$ 12$ an intrinsic meaning to linearization of equivariant (co)chain complexes, in the case when the CW-complex $X$ admits a cell structure with a minimal number of cells in each dimension.

Pick a basis $\left\{e_{1}, \ldots, e_{n}\right\}$ for $H_{1}=H_{1}(X, \mathbb{k})$, and identify the symmetric algebra $S=\operatorname{Sym}\left(H_{1}\right)$ with the polynomial ring $\mathbb{k}\left[e_{1}, \ldots, e_{n}\right]$. The universal Aomoto 
complex of $H^{*}=H^{*}(X, \mathbb{k})$ is the cochain complex of free $S$-modules,

$$
H^{0} \otimes_{\mathbb{k}} S \stackrel{D^{0}}{\longrightarrow} H^{1} \otimes_{\mathbb{k}} S \stackrel{D^{1}}{\longrightarrow} H^{2} \otimes_{\mathbb{k}} S \stackrel{D^{2}}{\longrightarrow} \cdots,
$$

with differentials $D(\alpha \otimes 1)=\sum_{i=1}^{n} e_{i}^{*} \cdot \alpha \otimes e_{i}$.

Theorem D. Let $X$ be a minimal $C W$-complex, and assume $\mathbb{k}=\mathbb{Z}$ or a field.

(1) Let $\nu: \pi \rightarrow G$ be an epimorphism. Then the linearization of the equivariant chain complex, $\left(C_{\bullet}\left(X, \mathbb{k} G_{\nu}\right), \tilde{\partial}_{\bullet}^{G}\right)$, is equal to the $E^{1}$-term of the equivariant spectral sequence, $\left(E^{1}\left(X, \mathbb{k} G_{\nu}\right), d_{G}^{1}\right)$.

(2) The linearization of the equivariant cochain complex of the universal abelian cover of $X$ coincides with the universal Aomoto complex of $H^{*}(X, \mathbb{k})$.

Part (1) - suitably interpreted with the aid of Theorem A-was proved in 7. Theorem 20], in the case when $\nu=\mathrm{id}$ and $\mathbb{k}=\mathbb{Z}$, and under the additional assumption that the cohomology ring $H^{*}(X, \mathbb{Z})$ is generated in degree one.

Part (2) generalizes [4, Theorem 1.2], valid only for complements of complex hyperplane arrangements, and $\mathbb{k}=\mathbb{C}$. Earlier results in this direction, also within the confines of arrangement theory, were obtained in [5] and [3]. The result in part (2) was recently proved by M. Yoshinaga [35], under an additional condition, satisfied by arrangement complements, but not by arbitrary minimal CW-complexes. Theorem D(2) shows that this condition is unnecessary, thereby answering a question raised by Yoshinaga in Remark 15 of the preprint version of 35].

\section{The Equivariant Chain COMPlex of a CW-COMPlex}

In this section, we review a well-known construction that goes back to J.H.C. Whitehead [34] and S. Eilenberg [10]: the chain complex of the universal cover of a cell complex $X$, with coefficients in a $\mathbb{k} \pi_{1}(X)$-module $M$. We start with some basic algebraic notions.

2.1. Associated graded rings. Let $R$ be a ring, and $J$ a two-sided ideal. The successive powers of $J$ determine a descending filtration on $R$, called the $J$-adic filtration. The associated graded ring, $\operatorname{gr}_{J}(R)$, is defined as

$$
\operatorname{gr}_{J}(R)=\bigoplus_{n \geq 0} J^{n} / J^{n+1}
$$

On homogeneous components, the multiplication map $\operatorname{gr}_{J}^{n}(R) \otimes \operatorname{gr}_{J}^{m}(R) \rightarrow \operatorname{gr}_{J}^{n+m}(R)$ is induced from multiplication in the ring $R$.

We will be mainly interested in the following situation. Let $\pi$ be a group, and let $\mathbb{k}$ be a commutative ring with unit 1 . The group ring of $\pi$, denoted $\mathbb{k} \pi$, consists of all finite linear combinations of group elements with coefficients in $\mathbb{k}$. Multiplication in $\mathbb{k} \pi$ is induced from the group operation on $\pi$; the unit (denoted by 1 ) is $1 \cdot \iota$, where $\iota$ is the identity of $\pi$. The augmentation ideal, $I=I_{\mathrm{k}} \pi$, is the kernel of the ring homomorphism $\epsilon: \mathbb{k} \pi \rightarrow \mathbb{k}, \sum n_{g} g \mapsto \sum n_{g}$; as a $\mathbb{k}$-module, $I$ is freely generated by the elements $g-1$, for $g \neq \iota$.

The augmentation ideal defines the $I$-adic filtration on $\mathbb{k} \pi$; let $\operatorname{gr}(\mathbb{k} \pi)=\operatorname{gr}_{I}(\mathbb{k} \pi)$ be the associated graded ring. Note that $\operatorname{gr}(\mathbb{k} \pi)$ is always generated as a ring in degree 1. Clearly, $\operatorname{gr}^{0}(\mathbb{k} \pi) \cong \mathbb{k}$. Moreover, the map $\pi \rightarrow I, g \mapsto g-1$ factors through an isomorphism $H_{1}(\pi, \mathbb{k}) \rightarrow I / I^{2}$ (see [17]). Thus,

$$
\operatorname{gr}^{1}(\mathbb{k} \pi) \cong H_{1}(\pi, \mathbb{k}) \text {. }
$$


As an example, consider the free abelian group $\pi=\mathbb{Z}^{n}$. The group ring $\mathbb{k} \mathbb{Z}^{n}$ can be identified with the Laurent polynomial ring $\mathbb{k}\left[t_{1}^{ \pm 1}, \ldots, t_{n}^{ \pm 1}\right]$, while $\operatorname{gr}\left(\mathbb{k} \mathbb{Z}^{n}\right)$ can be identified with $\mathbb{k}\left[x_{1}, \ldots, x_{n}\right]$, the polynomial ring in $n$ variables, via the map $t_{i}-1 \mapsto x_{i}$

2.2. Associated graded modules. A similar construction applies to modules: if $M$ is a left $\mathbb{k} \pi$-module, then $M$ is filtered by the submodules $\left\{I^{n} M\right\}_{n \geq 0}$. The associated graded object, $\operatorname{gr}_{I}(M)=\bigoplus_{n>0} I^{n} M / I^{n+1} M$, inherits a graded module structure over $\operatorname{gr}(\mathbb{k} \pi)$. This module is generated in degree 0 by the module of coinvariants, $M / I M$.

All these constructions are functorial. For example, if $\alpha: \pi \rightarrow \pi^{\prime}$ is a group homomorphism, then the linear extension to group rings, $\bar{\alpha}: \mathbb{k} \pi \rightarrow \mathbb{k} \pi^{\prime}$, is a ring map, preserving the respective $I$-adic filtrations. Thus, $\bar{\alpha}$ induces a degree 0 ring homomorphism, $\operatorname{gr}(\alpha): \operatorname{gr}(\mathbb{k} \pi) \rightarrow \operatorname{gr}\left(\mathbb{k} \pi^{\prime}\right)$.

Given a $\mathbb{k} \pi$-module $M$ and a $\mathbb{k} \pi^{\prime}$-module $M^{\prime}$, a $\mathbb{k}$-linear map $\phi: M \rightarrow M^{\prime}$ is said to be equivariant with respect to the ring map $\bar{\alpha}: \mathbb{k} \pi \rightarrow \mathbb{k} \pi^{\prime}$ if $\phi(g m)=\alpha(g) \phi(m)$, for all $g \in \pi$ and $m \in M$. Such a map $\phi$ preserves $I$-adic filtrations and thus induces a map $\operatorname{gr}(\phi): \operatorname{gr}_{I}(M) \rightarrow \operatorname{gr}_{I}\left(M^{\prime}\right)$, equivariant with respect to $\operatorname{gr}(\alpha)$.

Completely analogous considerations apply to right $\mathbb{k} \pi$-modules.

2.3. Chains on the universal cover. Let $X$ be a connected CW-complex with skeleta $\left\{X^{q}\right\}_{q \geq 0}$. Up to homotopy, we may assume $X$ has a single 0-cell, call it $e_{0}$, which we will take as the basepoint. Moreover, we may assume all attaching maps $\left(S^{q}, *\right) \rightarrow\left(X^{q}, e_{0}\right)$ are basepoint-preserving.

Fix a commutative ring $\mathbb{k}$ with unit, and denote by $C_{\bullet}(X, \mathbb{k})=\left(C_{q}(X, \mathbb{k}), \partial_{q}\right)_{q \geq 0}$ the cellular chain complex of $X$, with coefficients in $\mathbb{k}$. Recall that

$$
C_{q}(X, \mathbb{k})=H_{q}\left(X^{q}, X^{q-1}, \mathbb{k}\right)
$$

is a free $\mathbb{k}$-module, with basis indexed by the $q$-cells of $X$.

Let $p: \widetilde{X} \rightarrow X$ be the universal cover. The cell structure on $X$ lifts to a cell structure on $\tilde{X}$. Fixing a lift $\tilde{e}_{0} \in p^{-1}\left(e_{0}\right)$ identifies the fundamental group $\pi=$ $\pi_{1}\left(X, e_{0}\right)$ with the group of deck transformations of $\widetilde{X}$, which permute the cells. Therefore, we may view

$$
C_{\bullet}(\widetilde{X}, \mathbb{k})=\left(C_{q}(\widetilde{X}, \mathbb{k}), \tilde{\partial}_{q}\right)_{q \geq 0}
$$

as a chain complex of left-modules over the group ring $\mathbb{k} \pi$. We shall call $C_{\bullet}(\widetilde{X}, \mathbb{k})$ the equivariant chain complex of $X$ over $\mathbb{k}$.

Using the action of $\pi$ on $\widetilde{X}$, we may identify

$$
C_{q}(\tilde{X}, \mathbb{k}) \cong \mathbb{k} \pi \otimes_{\mathbb{k}} C_{q}(X, \mathbb{k})
$$

as left $\mathbb{k} \pi$-modules. Under this identification, a basis element of the form $1 \otimes e$ from the right hand side corresponds to the unique lift $\tilde{e}$ of the $q$-cell $e$ sending the basepoint $* \in D^{q}$ to $\tilde{e}_{0}$.

These constructions are functorial in the following sense. Suppose $f: X \rightarrow X^{\prime}$ is a map between connected $\mathrm{CW}$-complexes. By cellular approximation, we may assume $f$ respects the CW-structures; in particular, $f\left(e_{0}\right)=e_{0}^{\prime}$. Let $f_{*}: C_{\bullet}(X, \mathbb{k}) \rightarrow$ $C_{\bullet}\left(X^{\prime}, \mathbb{k}\right)$ be the induced map on cellular chain complexes, and let $f_{\sharp}: \pi \rightarrow \pi^{\prime}$ be the induced homomorphism on fundamental groups. By covering space theory, the map $f$ lifts to a cellular map, $\tilde{f}: \widetilde{X} \rightarrow \widetilde{X}^{\prime}$, uniquely specified by the requirement 
that $\tilde{f}\left(\tilde{e}_{0}\right)=\tilde{e}_{0}^{\prime}$. The induced chain map, $\tilde{f}_{*}: C_{\bullet}(\tilde{X}, \mathbb{k}) \rightarrow C_{\bullet}\left(\tilde{X}^{\prime}, \mathbb{k}\right)$, is equivariant with respect to the ring homomorphism $\bar{f}_{\sharp}: \mathbb{k} \pi \rightarrow \mathbb{k} \pi^{\prime}$.

2.4. The first differentials. Let $\epsilon: \mathbb{k} \pi \rightarrow \mathbb{k}$ be the augmentation homomorphism. Under identification (4), the induced homomorphism $p_{*}: C_{q}(\tilde{X}, \mathbb{k}) \rightarrow C_{q}(X, \mathbb{k})$ coincides with $\epsilon \otimes \mathrm{id}: \mathbb{k} \pi \otimes_{\mathbb{k}} C_{q}(X, \mathbb{k}) \rightarrow \mathbb{k} \otimes_{\mathbb{k}} C_{q}(X, \mathbb{k})$.

The first boundary map, $\tilde{\partial}_{1}$, is easy to write. Let $e_{1}$ be a 1-cell of $X$. Recall that we assume $X$ has a single 0 -cell, $e_{0}$; thus, $e_{1}$ is a loop at $e_{0}$, representing an element $x=\left[e_{1}\right] \in \pi$. Let $\tilde{e}_{1}$ be the lift of $e_{1}$ at $\tilde{e}_{0}$. Clearly, $\tilde{\partial}_{1}\left(\tilde{e}_{1}\right)=(x-1) \tilde{e}_{0}$. Thus, $\tilde{\partial}_{1}: \mathbb{k} \pi \otimes_{\mathbb{k}} C_{1}(X, \mathbb{k}) \rightarrow \mathbb{k} \pi \otimes_{\mathbb{k}} C_{0}(X, \mathbb{k})=\mathbb{k} \pi$ is given by

$$
\tilde{\partial}_{1}\left(1 \otimes e_{1}\right)=x-1 \text {. }
$$

The second boundary map, $\tilde{\partial}_{2}: \mathbb{k} \pi \otimes_{\mathbb{k}} C_{2}(X, \mathbb{k}) \rightarrow \mathbb{k} \pi \otimes_{\mathbb{k}} C_{1}(X, \mathbb{k})$, can be written by means of Fox derivatives [13. If $\left\{e_{1}^{i}\right\}_{i}$ are the 1-cells of $X$ and $e_{2}$ is a 2-cell, then

$$
\tilde{\partial}_{2}\left(1 \otimes e_{2}\right)=\sum_{i}\left(\frac{\partial r}{\partial x_{i}}\right)^{\phi} \otimes e_{1}^{i}
$$

where $r$ is the word in the free group $F$ on generators $x_{i}$, determined by the attaching map of the 2-cell, and $\bar{\phi}: \mathbb{k} F \rightarrow \mathbb{k} \pi$ is the extension to group rings of the projection map $\phi: F \rightarrow \pi$. As for the higher boundary maps $\tilde{\partial}_{q}, q>2$, there is no general procedure for computing them, except in certain very specific situations.

If $X$ is an Eilenberg-MacLane $K(\pi, 1)$ space, i.e., if $\widetilde{X}$ is contractible, then the augmented chain complex $\widetilde{C}_{\bullet}: C_{\bullet}(\widetilde{X}, \mathbb{k}) \rightarrow \mathbb{k}$ is a free $\mathbb{k} \pi$-resolution of the trivial module $\mathbb{k}$. For instance, if $T^{n}=K\left(\mathbb{Z}^{n}, 1\right)$ is the $n$-torus, then $\widetilde{C} \bullet$ is the Koszul complex over the ring $\mathbb{k} \mathbb{Z}^{n}=\mathbb{k}\left[t_{1}^{ \pm 1}, \ldots, t_{n}^{ \pm 1}\right]$.

2.5. Building up $\mathbf{C W}$-complexes. Given a group $\pi$ and a matrix $D$ over $\mathbb{Z} \pi$, it is possible to construct a CW-complex $X$ having $D$ as a block in one of the equivariant boundary maps in $C_{\bullet}(\tilde{X}, \mathbb{Z})$.

Example 2.1. Let $X_{0}$ be a connected CW-complex, with fundamental group $\pi=$ $\pi_{1}\left(X_{0}, e_{0}\right)$, and denote by $X_{0} \vee S^{n}$ the wedge sum of $X_{0}$ with the $n$-sphere, $n \geq 2$. Note that $\pi_{1}\left(X_{0} \vee S^{n}\right)=\pi$. The inclusion $S^{n} \hookrightarrow X_{0} \vee S^{n}$ defines an element $\left[S^{n}\right] \in \pi_{n}\left(X_{0} \vee S^{n}\right)$.

Given an element $x$ in $\mathbb{Z} \pi$, construct a new CW-complex, $X$, by attaching an $(n+1)$-cell along a map $\phi_{x}: S^{n} \rightarrow X_{0} \vee S^{n}$ representing $x \cdot\left[S^{n}\right] \in \pi_{n}\left(X_{0} \vee S^{n}\right)$. Clearly, $\pi_{1}(X)=\pi_{1}\left(X_{0}\right)$, and $C_{\bullet}(\widetilde{X}, \mathbb{Z})=C_{\bullet}\left(\widetilde{X}_{0}, \mathbb{Z}\right) \oplus C(n, x)$, where $C(n, x)$ denotes the elementary chain complex $C_{n+1} \rightarrow C_{n}$, with a differential $\cdot x: \mathbb{Z} \pi \rightarrow \mathbb{Z} \pi$.

More generally, for any integer $n \geq 2$ and any $\mathbb{Z} \pi$-linear map $D:(\mathbb{Z} \pi)^{m} \rightarrow(\mathbb{Z} \pi)^{\ell}$, a construction much as above produces a CW-complex $X$, with $\pi_{1}(X)=\pi_{1}\left(X_{0}\right)$, and $C \bullet(\widetilde{X}, \mathbb{Z})=C \bullet\left(\widetilde{X}_{0}, \mathbb{Z}\right) \oplus C(n, D)$, where $C(n, D)$ denotes the chain complex concentrated in degrees $n+1$ and $n$, with a differential equal to $D$.

2.6. Coefficient modules. Let $X$ be a connected CW-complex with fundamental group $\pi$. Suppose $M$ is a right $\mathbb{k} \pi$-module. The cellular chain complex of $X$ with coefficients in $M$ is defined as

$$
\begin{aligned}
C \bullet(X, M) & =\left(C_{q}(X, M), \tilde{\partial}_{q}^{M}\right)_{q \geq 0} \\
& =\left(M \otimes_{\mathbb{k} \pi} C_{q}(\widetilde{X}, \mathbb{k}), \operatorname{id}_{M} \otimes_{\mathbb{k} \pi} \tilde{\partial}_{q}\right)_{q \geq 0} .
\end{aligned}
$$


In the particular case when $M$ is the free $\mathbb{k} \pi$-module of rank one, $C_{\bullet}(X, \mathbb{k} \pi)$ coincides with the equivariant chain complex $C \bullet(\widetilde{X}, \mathbb{k})$.

Similarly, if $M$ is a left $\mathbb{k} \pi$-module, define the cellular cochain complex of $X$ with coefficients in $M$ as

$$
\begin{aligned}
C^{\bullet}(X, M) & =\left(C^{q}(X, M), \tilde{\delta}_{M}^{q}\right)_{q \geq 0} \\
& =\left(\operatorname{Hom}_{\mathbb{k} \pi}\left(C_{q}(\widetilde{X}, \mathbb{k}), M\right), \operatorname{Hom}_{\mathbb{k} \pi}\left(\tilde{\partial}_{q}, M\right)\right)_{q \geq 0} .
\end{aligned}
$$

Example 2.2. Let $\mathbb{k}$ be a field, and let $\rho: \pi \rightarrow \operatorname{GL}(n, \mathbb{k})$ be a linear representation of $\pi$. Then $M=\mathbb{k}^{n}$ acquires a right module structure over $\mathbb{k} \pi$, via $m g=\rho\left(g^{-1}\right)(m)$; such a module is called a rank $n$ local system on $X$, with monodromy $\rho$. We write $H_{*}\left(X,{ }_{\rho} \mathbb{k}^{n}\right):=H_{*}(C \bullet(X, M))$ for the homology of $X$ with coefficients in this local system. When $n=1$, there is no need to turn a representation into an antirepresentation; in this case, we simply define $H_{*}\left(X, \mathbb{k}_{\rho}\right)$ to be $H_{*}(C \bullet(X, M))$, where $M=\mathbb{k}$ is viewed as a right $\mathbb{k} \pi$-module, via $m g=\rho(g)(m)$.

Example 2.3. Let $Y \rightarrow X$ be a Galois cover, with a group of deck transformations $G$ and classifying map $\nu: \pi \rightarrow G$. The cell structure on $X$ lifts in standard fashion to a cell structure on $Y$. Let $\mathbb{k} G_{\nu}$ denote the group-ring of $G$, viewed as a right $\mathbb{k} \pi$ module, via $h \cdot g=h \nu(g)$. Similarly, let ${ }_{\nu} \mathbb{k} G$ denote the same group-ring, viewed as a left $\mathbb{k} \pi$-module, via $g \cdot h=\nu(g) h$. Then $C_{\bullet}(Y, \mathbb{k})=C_{\bullet}\left(X, \mathbb{k} G_{\nu}\right)$, as chain complexes of left $\mathbb{k} G$-modules, and $C^{\bullet}(Y, \mathbb{k})=C^{\bullet}\left(X,{ }_{\nu} \mathbb{k} G\right)$, as cochain complexes of right $\mathbb{k} G$-modules.

Notable is the case of the universal abelian cover, $X^{\mathrm{ab}} \rightarrow X$, defined by the abelianization map, ab: $\pi \rightarrow \pi_{\mathrm{ab}}$. The homology groups $H_{q}\left(X^{\mathrm{ab}}, \mathbb{k}\right)$, viewed as modules over the ring $\Lambda=\mathbb{k} \pi_{\mathrm{ab}}$, are called the Alexander invariants of $X$, while the boundary maps $\widetilde{\partial}_{q}^{\mathrm{ab}}=\mathrm{id}_{\Lambda} \otimes_{\mathrm{k} \pi} \widetilde{\partial}_{q}$ are called the Alexander matrices of $X$.

\section{The EQUIVARIANT SPECTRAL SEQUENCE}

We now set up the spectral sequence associated to the $I$-adic filtration on the equivariant chain complex $C \cdot(X, M)$ and analyze some of its properties.

3.1. A spectral sequence. We use 2] and 31] as standard references for spectral sequences. Given an increasing filtration $F_{\bullet}=\left\{F_{-n}\right\}_{n \geq 0}$ on a chain complex $C_{\bullet}=\left(C_{q}, \partial_{q}\right)$ over a coefficient ring $\mathbb{k}$, there is a spectral sequence $E^{\bullet}=$ $\left\{E_{s, t}^{r}, d^{r}\right\}_{r \geq 1}$. The $E^{1}$ term is defined as $E_{s, t}^{1}=H_{s+t}\left(F_{s} / F_{s-1}\right)$, while the $d^{1}$ differential is the boundary operator in the homology exact sequence associated to the triple $\left(F_{s}, F_{s-1}, F_{s-2}\right)$. Each term $E^{r}$ is a bigraded $\mathbb{k}$-module, the differentials $d^{r}$ have bidegree $(-r, r-1)$, and $E^{r+1}=H\left(E^{r}, d^{r}\right)$.

Now let $X$ be a connected CW-complex as in $\$ 2.3$ with fundamental group $\pi=\pi_{1}(X)$ and augmentation ideal $I=I_{\mathbb{k}}(\pi)$. Let $M$ be a right $\mathbb{k} \pi$-module, and let $C_{\bullet}(X, M)$ be the cellular chain complex of $X$ with coefficients in $M$.

The $I$-adic filtration on $M$ yields a descending filtration, $F^{0} \supset F^{1} \supset F^{2} \supset \cdots$, on $C \bullet(X, M)$. The $n$-th term of this filtration is given by

$$
F^{n}(C \bullet(X, M))=M \cdot I^{n} \otimes_{\mathbb{k} \pi} C_{\bullet}(\tilde{X}, \mathbb{k}) .
$$

Clearly, the differentials $\tilde{\partial}^{M}$ of $C_{\bullet}(X, M)$ preserve this filtration. Set $F_{-n}=F^{n}$. Then $F_{\bullet}=\left\{F_{-n}\right\}_{n \geq 0}$ is an increasing filtration on $C \bullet(X, M)$, bounded above by $F_{0}=C_{\bullet}(X, M)$. 
Definition 3.1. The equivariant spectral sequence of the CW-complex $X$, with coefficients in the $\mathbb{k} \pi_{1}(X)$-module $M$, is the spectral sequence associated to the $I$-adic filtration $F_{\bullet}$ on the chain complex $C \bullet(X, M)$,

$$
\left\{E^{r}(X, M), d^{r}\right\}_{r \geq 0}
$$

with differentials $d^{r}: E_{s, t}^{r} \rightarrow E_{s-r, t+r-1}^{r}$.

In order to analyze this spectral sequence, we need some preliminary facts.

3.2. The associated graded chain complex. Under the identification from (4), the terms of the chain complex (7) can be written as

$$
C_{q}(X, M)=M \otimes_{\mathbb{k}} C_{q}(X, \mathbb{k}) .
$$

The $I$-adic filtration on this chain complex is then given by

$$
F^{n}(C \bullet(X, M))=M \cdot I^{n} \otimes_{\mathbb{k}} C \bullet(X, \mathbb{k}) .
$$

Clearly, $F^{n} / F^{n+1}=\operatorname{gr}^{n}(M) \otimes_{\mathbb{k}} C \cdot(X, \mathbb{k})$, where $\operatorname{gr}(M)=\bigoplus_{n>0} M I^{n} / M I^{n+1}$. Now recall that the boundary maps in $C \cdot(X, M)$ have the form $\tilde{\partial}^{M}=\operatorname{id}_{M} \otimes_{\mathbb{k} \pi} \tilde{\partial}$.

Lemma 3.2. For each $q \geq 0$, we have $\operatorname{gr}\left(\operatorname{id}_{M} \otimes_{\mathbb{k} \pi} \tilde{\partial}_{q}\right)=\operatorname{id}_{\operatorname{gr}(M)} \otimes_{\mathbb{k}} \partial_{q}$.

Proof. Let $e$ be a $q$-cell of $X$. Then

$$
p_{*} \tilde{\partial}_{q}(1 \otimes e)=\partial_{q} p_{*}(1 \otimes e)=\partial_{q}(e)=p_{*}\left(1 \otimes \partial_{q} e\right) .
$$

Hence, $\tilde{\partial}_{q}(1 \otimes e)-1 \otimes \partial_{q}(e)$ belongs to $I \otimes_{\mathbb{k}} C_{q-1}(X, \mathbb{k})$.

Now let $m$ be an arbitrary element in $M \cdot I^{n}$. By the above,

$$
\left(\operatorname{id}_{M} \otimes_{\mathbb{k} \pi} \tilde{\partial}_{q}\right)(m \otimes e)-m \otimes \partial_{q}(e) \in M \cdot I^{n+1} \otimes_{\mathbb{k}} C_{q-1}(X, \mathbb{k}) .
$$

The conclusion follows at once.

It follows that the graded chain complex associated to filtration (11) has the form

$$
\operatorname{gr}(C \bullet(X, M))=\left(\operatorname{gr}(M) \otimes_{\mathbb{k}} C_{q}(X, \mathbb{k}), \operatorname{id}_{\operatorname{gr}(M)} \otimes \partial_{q}\right)_{q \geq 0} .
$$

This chain complex is typically much easier to handle than the equivariant chain complex (7). Here is an illustration.

Example 3.3. Consider a local system $M$ as in Example 2.2. Suppose there is an element $g \in \pi$ such that $\rho(g)$ does not admit 1 as an eigenvalue - this happens whenever $M$ is a non-trivial, rank 1 local system. Then clearly $M I=M$. Thus, $\operatorname{gr}(M)=0$ and $\operatorname{gr}\left(C_{\bullet}(X, M)\right)$ is the zero complex in this instance.

Remark 3.4. In the case when $M=\mathbb{k} \pi$, the $I$-adic filtration on $C \bullet(X, M)=$ $C_{\bullet}(\widetilde{X}, \mathbb{k})$ is simply $C_{\bullet}(\widetilde{X}, \mathbb{k}) \supseteq I \cdot C_{\bullet}(\widetilde{X}, \mathbb{k}) \supseteq \cdots \supseteq I^{n} \cdot C_{\bullet}(\widetilde{X}, \mathbb{k}) \supseteq \cdots$, while the associated graded chain complex takes the form

$$
\operatorname{gr}(C \cdot(\widetilde{X}, \mathbb{k}))=\left(\operatorname{gr}(\mathbb{k} \pi) \otimes_{\mathbb{k}} C_{q}(X, \mathbb{k}), \operatorname{id}_{\operatorname{gr}(\mathbb{k} \pi)} \otimes \partial_{q}\right)_{q \geq 0} .
$$

Notice that the differentials in this chain complex are $\operatorname{gr}(\mathbb{k} \pi)$-linear. 
3.3. The first pages. The $E^{0}$ term of the equivariant spectral sequence of $X$ with coefficients in $M$ is defined in the usual manner, as the associated graded of the filtration $F_{\bullet}$ on $C_{\bullet}(X, M)$. Using (12), we find that

$$
E_{-p, q}^{0}(X, M)=\operatorname{gr}^{p}(M) \otimes_{\mathbb{k}} C_{q-p}(X, \mathbb{k}),
$$

if $p \geq 0$ and $q \geq p$, and otherwise $E_{-p, q}^{0}(X, M)=0$. Hence, the spectral sequence is concentrated in the second quadrant. Under this identification, and as a consequence of Lemma 3.2, the differential $d^{0}: E_{-p, q}^{0}(X, M) \rightarrow E_{-p, q-1}^{0}(X, M)$ takes the form $d^{0}=\mathrm{id} \otimes \partial$. Hence,

$$
E_{-p, q}^{1}(X, M)=H_{q-p}\left(X, \operatorname{gr}^{p}(M)\right) .
$$

3.4. Functoriality properties. Let $f:\left(X, e_{0}\right) \rightarrow\left(X^{\prime}, e_{0}^{\prime}\right)$ be a cellular map, and suppose $\phi: M \rightarrow M^{\prime}$ is equivariant with respect to $f_{\sharp}$. The resulting $\mathbb{k}$-linear map,

$$
\phi \otimes \tilde{f}_{*}: C_{\bullet}(X, M)=M \otimes_{\mathbb{k} \pi} C_{\bullet}(\widetilde{X}, \mathbb{k}) \rightarrow M^{\prime} \otimes_{\mathbb{k} \pi^{\prime}} C_{\bullet}\left(\widetilde{X}^{\prime}, \mathbb{k}\right)=C_{\bullet}\left(X^{\prime}, M^{\prime}\right),
$$

is a chain map, preserving $I$-adic filtrations. Consequently, $\phi \otimes \tilde{f}_{*}$ induces a morphism between the corresponding $I$-adic spectral sequences,

$$
E^{r}\left(\phi \otimes \tilde{f}_{*}\right): E^{r}(X, M) \rightarrow E^{r}\left(X^{\prime}, M^{\prime}\right) .
$$

In particular, $\phi \otimes \tilde{f}_{*}$ induces a chain map between the associated graded chain complexes,

$$
\operatorname{gr}\left(\phi \otimes \tilde{f}_{*}\right): \operatorname{gr}(M) \otimes_{\mathbb{k}} C_{\bullet}(X, \mathbb{k}) \rightarrow \operatorname{gr}\left(M^{\prime}\right) \otimes_{\mathbb{k}} C_{\bullet}\left(X^{\prime}, \mathbb{k}\right) .
$$

Lemma 3.5. For each $q \geq 0$, we have $\operatorname{gr}\left(\phi \otimes \tilde{f}_{q}\right)=\operatorname{gr}(\phi) \otimes f_{q}$.

Proof. It is readily seen that, under the identification $\operatorname{gr}^{0}(\mathbb{k} \pi)=\mathbb{k}$, the $\operatorname{map} \operatorname{gr}^{0}\left(\tilde{f}_{q}\right)$ corresponds to the map $f_{q}: C_{q}(X, \mathbb{k}) \rightarrow C_{q}\left(X^{\prime}, \mathbb{k}\right)$.

Let $m$ be an element of $M I^{n}$, and let $e$ be a $q$-cell of $X$, with lift $\tilde{e}$. By the above,

$$
\tilde{f}_{q}(\tilde{e})-1 \otimes f_{q}(e) \in I^{\prime} \cdot C_{q}\left(\tilde{X}^{\prime}, \mathbb{k}\right) .
$$

Hence, $\left(\phi \otimes \tilde{f}_{q}\right)(m \otimes \tilde{e})=\phi(m) \otimes \tilde{f}_{q}(\tilde{e})$ equals $\phi(m) \otimes f_{q}(e)$ modulo $F^{\prime n+1}$. The conclusion follows.

As a simple example, take $f=\operatorname{id}_{X}$. Then any morphism $\phi: M \rightarrow M^{\prime}$ of right $\mathbb{k} \pi$-modules induces a morphism of spectral sequences, $E^{\bullet}(\phi): E^{\bullet}(X, M) \rightarrow$ $E^{\bullet}\left(X, M^{\prime}\right)$, with $E^{0}(\phi)=\operatorname{gr}(\phi) \otimes$ id.

3.5. Homotopy invariance. Let $f:\left(X, e_{0}\right) \rightarrow\left(X^{\prime}, e_{0}^{\prime}\right)$ be a cellular homotopy equivalence, and let $M$ be a right $\mathbb{k} \pi$-module. Use the isomorphism $f_{\sharp}: \pi \rightarrow \pi^{\prime}$ to view $M$ as a right $\mathbb{k} \pi^{\prime}$-module.

Corollary 3.6. The spectral sequences $\left\{E^{r}(X, M)\right\}_{r \geq 1}$ and $\left\{E^{r}\left(X^{\prime}, M\right)\right\}_{r \geq 1}$ are isomorphic.

Proof. View $\operatorname{id}_{M}$ as an equivariant map with respect to $f_{\sharp}$. Using Lemma 3.5 and identification (15), we see that the map $E^{1}\left(\operatorname{id}_{M} \otimes \tilde{f}_{*}\right)$ coincides with the induced homomorphism $H_{*}(f): H_{*}(X, \operatorname{gr}(M)) \rightarrow H_{*}\left(X^{\prime}, \operatorname{gr}(M)\right)$. Hence, the maps $E^{r}\left(\operatorname{id}_{M} \otimes \tilde{f}_{*}\right)$ are isomorphisms, for all $r \geq 1$.

Corollary 3.7. Let $X$ be a path-connected topological space having the homotopy type of a $C W$-complex, and let $M$ be a right $\mathbb{k} \pi_{1}(X)$-module. Then there is a welldefined second quadrant $I$-adic spectral sequence $\left\{E^{r}(X, M), d^{r}\right\}$, starting at $r=1$. 


\section{IDENTIFYING The $d^{1}$ DIFFERENTIAL}

Throughout this section, $X$ is a $\mathrm{CW}$-complex with a single 0 -cell $e_{0}$ and with basepoint-preserving attaching maps, $\pi=\pi_{1}\left(X, e_{0}\right)$ is the fundamental group, and $C \cdot(X, M)$ is the cellular chain complex, with coefficients in a right $\mathbb{k} \pi$-module $M$. Our goal is to identify the first page of the equivariant spectral sequence of $X$ in terms of the coalgebra structure of $H_{*}(X, \mathbb{k})$ and the module structure of $M$.

4.1. The first page. Let $\left\{E^{r}(X, M), d^{r}\right\}_{r \geq 1}$ be the spectral sequence from Definition 3.1. We wish to analyze the differentials

$$
d^{1}: E_{-p, q}^{1}(X, M) \rightarrow E_{-p-1, q}^{1}(X, M),
$$

for all $p \geq 0$ and $q \geq p$. Schematically, the $E^{1}$ page looks like

$$
\begin{array}{r}
E_{-2,2}^{1} \stackrel{d^{1}}{\longleftarrow} E_{-1,2}^{1} \stackrel{\stackrel{d^{1}}{\longleftarrow} E_{0,2}^{1}}{E_{-1,1}^{1} \stackrel{d^{1}}{\longleftarrow} E_{0,1}^{1}} \\
E_{0,0}^{1} .
\end{array}
$$

To identify these maps in terms of cohomological data, we need to assume the following: either $\mathbb{k}=\mathbb{Z}$ and $H_{*}(X, \mathbb{Z})$ is torsion-free, or $\mathbb{k}$ is a field. Using this assumption, formula (15), and the Universal Coefficients Theorem, we find

$$
E_{-p, q}^{1}(X, M)=\operatorname{gr}^{p}(M) \otimes_{\mathbb{k}} H_{q-p}(X, \mathbb{k}),
$$

for each $p \geq 0$ and $q \geq p$.

The $d^{1}$ differentials enjoy the following naturality property. Suppose $f: X \rightarrow$ $X^{\prime}$ is a cellular map and $\phi: M \rightarrow M^{\prime}$ is a morphism of modules, equivariant with respect to $\bar{f}_{\sharp}: \mathbb{k} \pi_{1}(X) \rightarrow \mathbb{k} \pi_{1}\left(X^{\prime}\right)$. From Lemmas 3.2 and 3.5, we deduce $E^{1}\left(\phi \otimes \tilde{f}_{*}\right)=\operatorname{gr}(\phi) \otimes H_{*}(f)$. Using the naturality property from (17), we obtain a commuting diagram,

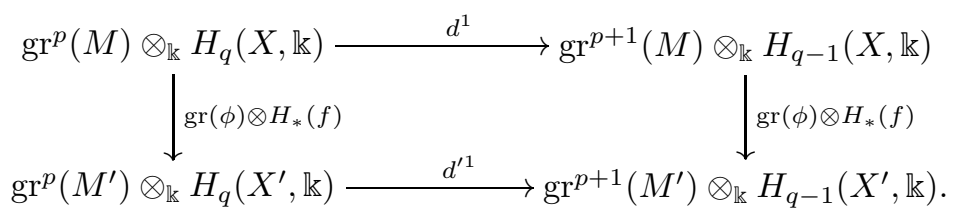

4.2. Cohomological interpretation. The main result of this section is the following theorem, which identifies the differentials on the $E^{1}$ page in terms of the comultiplication in $H_{*}(X, \mathbb{k})$ and the $\mathbb{k} \pi$-module structure on $M$, given by the multiplication map, $\mu_{M}: M \otimes_{\mathbb{k}} \mathbb{k} \pi \rightarrow M, m \otimes g \mapsto m g$. Fix integers $p \geq 0$ and $q \geq 1$.

Theorem 4.1. Let $X$ be a connected $C W$-complex, and $M$ a right $\mathbb{k} \pi$-module. Assume either $\mathbb{k}=\mathbb{Z}$ and $H_{*}(X, \mathbb{Z})$ is torsion-free, or $\mathbb{k}$ is a field. Then the differential $d^{1}: E_{-p, p+q}^{1}(X, M) \rightarrow E_{-p-1, p+q}^{1}(X, M)$ can be decomposed as

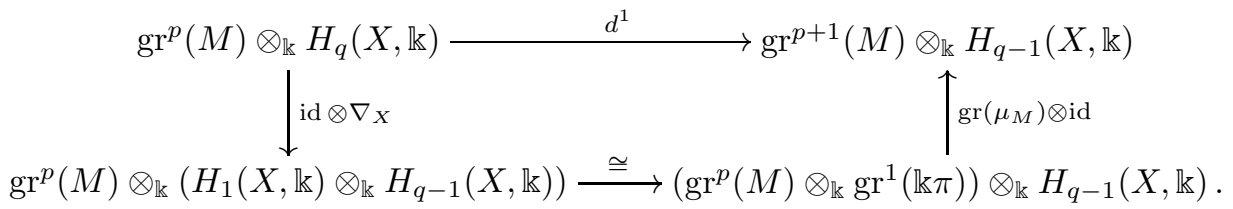


Here, $\nabla_{X}$ is the comultiplication map, defined as the composite

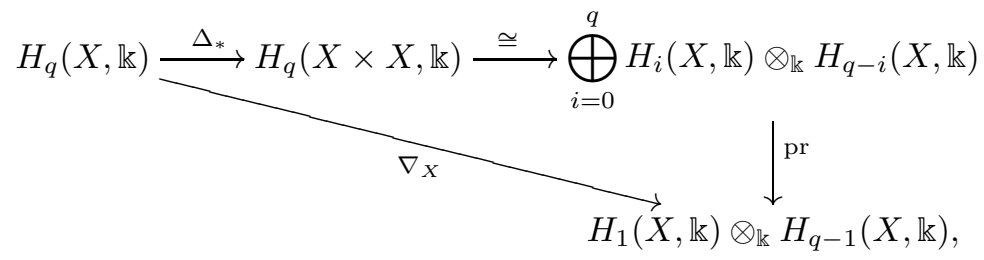

where the first arrow is the homomorphism induced by the diagonal map, the second arrow is the Künneth isomorphism, and the third arrow is the projection onto the direct summand. Under the identification $H_{i}(X, \mathbb{k})^{*} \cong H^{i}(X, \mathbb{k})$, the map $\nabla_{X}$ is the dual of the cup-product map $\bigcup_{X}: H^{1}(X, \mathbb{k}) \otimes_{\mathbb{k}} H^{q-1}(X, \mathbb{k}) \rightarrow H^{q}(X, \mathbb{k})$, provided $X$ is a finite-type $\mathrm{CW}$-complex.

The proof of Theorem 4.1 will occupy the rest of this section.

4.3. Reducing to the case $M=\mathbb{k} \pi$. Using the functoriality of the spectral sequence with respect to coefficient modules, we first reduce the proof to the case when $M$ is a free $\mathbb{k} \pi$-module of rank 1 .

Lemma 4.2. If the conclusion of Theorem 4.1 holds for the coefficient module $\mathbb{k} \pi$, then it holds for any coefficient module $M$.

Proof. Let $\psi: N \rightarrow M$ be a homomorphism of right $\mathbb{k} \pi$-modules. Write $H_{q}=$ $H_{q}(X, \mathbb{k})$, and $\otimes=\bigotimes_{\mathbb{k}}$. We then have the following cube diagram:

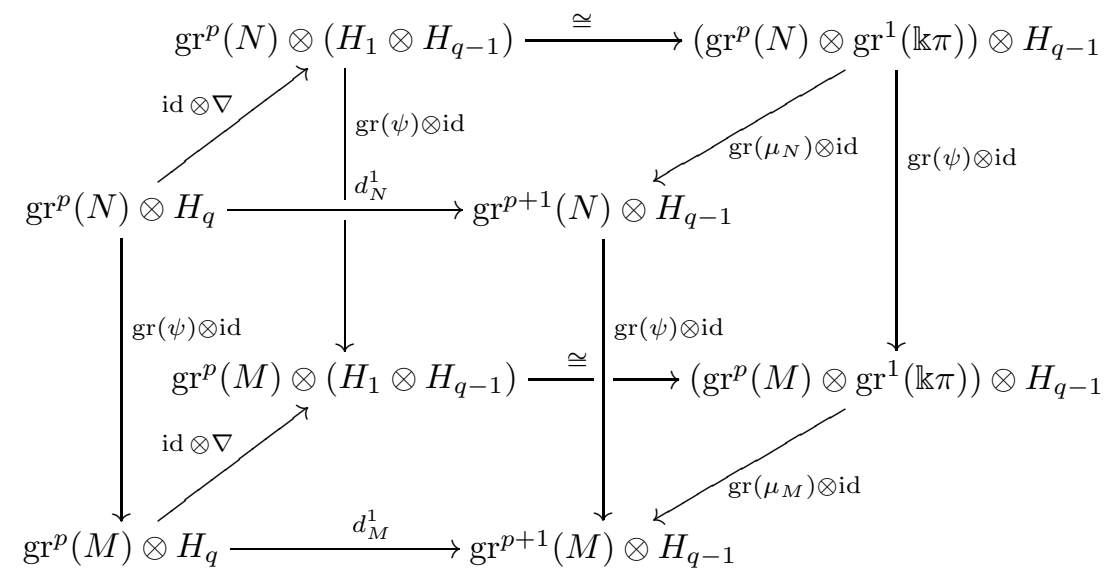

All side squares of the cube commute: the front one by naturality of $d^{1}$, as explained in (21), the right one by functoriality of gr, and the other two for obvious reasons.

Now suppose the top square commutes for $N=\mathbb{k} \pi$. Let $[x] \otimes h$ be an additive generator of $\operatorname{gr}^{p}(M) \otimes H_{q}$, where $x=y a$, with $y \in M$ and $a \in I^{p}$. Let $\psi: \mathbb{k} \pi \rightarrow M$ be the $\mathbb{k} \pi$-linear map sending the unit of $\pi$ to $y$. Chasing the cube diagram, we see that the bottom square commutes, with $[x] \otimes h=(\operatorname{gr}(\psi) \otimes \mathrm{id})([a] \otimes h)$ as input. Hence, the bottom square commutes.

4.4. The case $M=\mathbb{k} \pi$. The $E^{1}$-term of the $I$-adic spectral sequence of $X$ with coefficients in $\mathbb{k} \pi$,

$$
E^{1}(X, \mathbb{k} \pi)=\operatorname{gr}(\mathbb{k} \pi) \otimes_{\mathbb{k}} H_{*}(X, \mathbb{k})
$$


is a left $\operatorname{gr}(\mathbb{k} \pi)$-module, freely generated by a $\mathbb{k}$-basis for $1 \otimes H_{*}(X, \mathbb{k})$. We want to show

$$
d^{1}=\left(\operatorname{gr}\left(\mu_{\mathbb{k} \pi}\right) \otimes \mathrm{id}\right) \circ\left(\mathrm{id} \otimes \nabla_{X}\right) .
$$

Clearly, the map on the right side is $\operatorname{gr}(\mathbb{k} \pi)$-linear. In the next lemma, we verify that $d^{1}$ is $\operatorname{gr}(\mathbb{k} \pi)$-linear, too (a more general result will be proved in Lemma 6.2).

Lemma 4.3. The differential $d^{1}: E_{-p, p+q}^{1}(X, \mathbb{k} \pi) \rightarrow E_{-p-1, p+q}^{1}(X, \mathbb{k} \pi)$ is $\operatorname{gr}(\mathbb{k} \pi)$ linear.

Proof. Let $F^{s}=I^{s} C_{\bullet}(\widetilde{X}, \mathbb{k})$ be the $I$-adic filtration on $C_{\bullet}(X, \mathbb{k} \pi)$. By definition, the differential $d^{1}$ is the connecting homomorphism in the homology exact sequence of

$$
0 \longrightarrow F^{s+1} / F^{s+2} \longrightarrow F^{s} / F^{s+2} \longrightarrow F^{s} / F^{s+1} \longrightarrow 0 \text {. }
$$

Let $z \in C_{q}(X, \mathbb{k})$ such that $\partial_{q}(z)=0$. Then $d^{1}(1 \otimes[z]) \equiv \tilde{\partial}(1 \otimes z), \bmod F^{2}$. More generally, if $x \in I^{p}$, then $d^{1}([x] \otimes[z]) \equiv \tilde{\partial}(x \otimes z), \bmod F^{p+2}$. On the other hand, $\tilde{\partial}(x \otimes z)=x \tilde{\partial}(1 \otimes z)$. Combining these formulas implies $d^{1}([x] \otimes[z])=$ $[x] d^{1}(1 \otimes[z])$. Clearly, this implies the claim of the lemma.

Thus, to verify (22), it is sufficient to check equality on free $\operatorname{gr}(\mathbb{k} \pi)$-generators, i.e., on a $\mathbb{k}$-basis for $1 \otimes H_{*}(X, \mathbb{k})$. In other words, to identify the differentials on the $E^{1}$ page, we only need to show that, upon identifying $E_{0, q}^{1}=H_{q}(X, \mathbb{k})$ and $E_{-1, q}^{1}=H_{1}(X, \mathbb{k}) \otimes_{\mathbb{k}} H_{q-1}(X, \mathbb{k})$, the map $d^{1}: E_{0, q}^{1} \rightarrow E_{-1, q}^{1}$ coincides with the comultiplication map $\nabla_{X}$, for all $q \geq 1$.

4.5. Further reductions. Clearly, we may assume $X$ is a finite $\mathrm{CW}$-complex. Indeed, an arbitrary homology class in $H_{q}(X, \mathbb{k})$ is represented by a cycle supported on a finite subcomplex of $X$. Dualizing, we are left with proving the following proposition.

Proposition 4.4. Let $X$ be a connected, finite-type $C W$-complex, and assume either $\mathbb{k}=\mathbb{Z}$ and $H_{*}(X, \mathbb{Z})$ is torsion-free, or $\mathbb{k}$ is a field. Then, the dual $\delta^{1}=$ $\left(d^{1}\right)^{*}:\left(E_{-1, q}^{1}\right)^{*} \rightarrow\left(E_{0, q}^{1}\right)^{*}$ coincides with the cup-product map $\bigcup_{X}: H^{1}(X, \mathbb{k}) \otimes_{\mathbb{k}}$ $H^{q-1}(X, \mathbb{k}) \rightarrow H^{q}(X, \mathbb{k})$.

In the above, we may suppose $\mathbb{k}$ is actually a prime field. Indeed, in the first case, both $\delta^{1}$ and $\bigcup_{X}$ are defined over $\mathbb{Z}$, and $H^{*}(X, \mathbb{Z})$ injects into $H^{*}(X, \mathbb{Q})$, so we may replace $\mathbb{k}=\mathbb{Z}$ by $\mathbb{Q}$. In the second case, both maps are defined over the prime field of $\mathbb{k}$, so we may replace $\mathbb{k}$ by its prime field.

We will reduce the proof to a special class of spaces, using the functoriality properties of the spectral sequence. Let us first state those properties, in the form we will need.

Let $f: X \rightarrow X^{\prime}$ be a cellular map, and consider diagram (21) for the morphism $\phi=\bar{f}_{\sharp}: \mathbb{k} \pi \rightarrow \mathbb{k} \pi^{\prime}$. In degree $p=0$, this diagram simplifies to

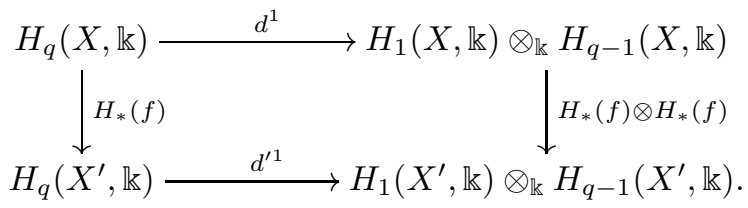


Dualizing, and writing $f^{*}=H^{*}(f)$, we obtain the commuting diagram

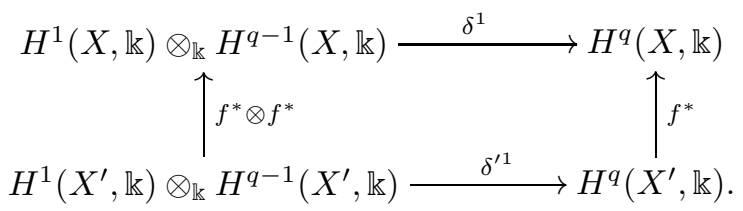

4.6. A computation with Eilenberg-MacLane spaces. Recall that $\mathbb{k}$ is a prime field. Let $R=\mathbb{Z}$ if $\mathbb{k}=\mathbb{Q}$, and $R=\mathbb{Z}_{p}$ if $\mathbb{k}=\mathbb{F}_{p}$. For an integer $r \geq 1$, let $K_{r}$ be an Eilenberg-MacLane space of type $K(R, r)$. We may assume $K_{r}$ is a finite-type CW-complex, obtained from $S^{r}$ by attaching cells of dimension $r+1$ and higher,

$$
K_{r}=e_{0} \cup e_{r} \cup e_{r+1}^{1} \cup \cdots \cup e_{r+1}^{m} \cup \cdots .
$$

Let $\llbracket e_{r} \rrbracket \in H_{r}\left(K_{r}, R\right)$ be the homology class represented by the $r$-cell, and let $\llbracket e_{r} \rrbracket^{*} \in H^{r}\left(K_{r}, \mathbb{k}\right)$ be its dual over $\mathbb{k}$.

Now let $K_{r}^{\prime}$ be another copy of $K_{r}$, with cells $e_{j}^{\prime}$, and consider the product CW-complex $K=K_{1} \times K_{r}^{\prime}$. Let $\mathrm{pr}$ and $\mathrm{pr}^{\prime}$ be the projections of $K$ onto the two factors. Define cohomology classes $u_{1}=\operatorname{pr}^{*}\left(\llbracket e_{1} \rrbracket^{*}\right) \in H^{1}(K, \mathbb{k})$ and $u_{r}^{\prime}=$ $\operatorname{pr}^{* *}\left(\llbracket e_{r}^{\prime} \rrbracket^{*}\right) \in H^{r}(K, \mathbb{k})$.

Lemma 4.5. For the $C W$-complex $K$ defined above, $\delta^{1}\left(u_{1} \otimes u_{r}^{\prime}\right)=u_{1} \cup u_{r}^{\prime}$.

Proof. Consider the map $\delta^{1}: H^{1}(K) \otimes H^{r}(K) \rightarrow H^{r+1}(K)$, with $\mathbb{k}$ coefficients. By Kronecker duality, we only need to verify

$$
\left\langle d^{1}(z), u_{1} \otimes u_{r}^{\prime}\right\rangle=\left\langle z, u_{1} \cup u_{r}^{\prime}\right\rangle,
$$

for all $z \in H_{r+1}(K)$. By the Künneth formula,

$H_{r+1}(K)=\left(H_{0}\left(K_{1}\right) \otimes H_{r+1}\left(K_{r}^{\prime}\right)\right) \oplus\left(H_{1}\left(K_{1}\right) \otimes H_{r}\left(K_{r}^{\prime}\right)\right) \oplus\left(H_{r+1}\left(K_{1}\right) \otimes H_{0}\left(K_{r}^{\prime}\right)\right)$.

By construction, $u_{1} \cup u_{r}^{\prime}=\llbracket e_{1} \rrbracket^{*} \times \llbracket e_{r}^{\prime} \rrbracket^{*}$. It follows that $\left\langle z, u_{1} \cup u_{r}^{\prime}\right\rangle=1$ if $z=$ $\llbracket e_{1} \rrbracket \times \llbracket e_{r}^{\prime} \rrbracket$ and vanishes if $z$ belongs to one of the other two summands in (26).

Similarly, the left hand side of (25) vanishes if $z$ belongs to one of those two summands; this follows from the naturality of $d^{1}$, as expressed in (23).

Now suppose $r>1$. Note that $\pi_{1}\left(K_{1}\right)$ is a cyclic group, generated by $\left[e_{1}\right]$, whereas $\pi_{1}\left(K_{r}^{\prime}\right)=0$. Moreover, $\widetilde{K}=\widetilde{K}_{1} \times K_{r}^{\prime}$. We compute that

$$
\tilde{\partial}\left(\tilde{e}_{1} \times e_{r}^{\prime}\right)=\tilde{\partial}\left(\tilde{e}_{1}\right) \times e_{r}^{\prime}-\tilde{e}_{1} \times \partial\left(e_{r}^{\prime}\right)=\left(\left[e_{1}\right]-1\right) \tilde{e}_{0} \times e_{r}^{\prime},
$$

and so $d^{1}\left(\llbracket e_{1} \times e_{r}^{\prime} \rrbracket\right)=\llbracket e_{1} \times e_{0}^{\prime} \rrbracket \otimes \llbracket e_{0} \times e_{r}^{\prime} \rrbracket$. It follows that $\left\langle d^{1}\left(\llbracket e_{1} \times e_{r}^{\prime} \rrbracket\right), u_{1} \otimes u_{r}^{\prime}\right\rangle=1$.

If $r=1$, then $\widetilde{K}=\widetilde{K}_{1} \times \widetilde{K}_{1}^{\prime}$. We compute that

$$
\tilde{\partial}\left(\tilde{e}_{1} \times \tilde{e}_{1}^{\prime}\right)=\tilde{\partial}\left(\tilde{e}_{1}\right) \times \tilde{e}_{1}^{\prime}-\tilde{e}_{1} \times \tilde{\partial}\left(\tilde{e}_{1}^{\prime}\right)=\left(\left[e_{1}\right]-1\right) \tilde{e}_{0} \times \tilde{e}_{1}^{\prime}-\left(\left[e_{1}^{\prime}\right]-1\right) \tilde{e}_{1} \times \tilde{e}_{0}^{\prime},
$$

and so $d^{1}\left(\llbracket e_{1} \times e_{1}^{\prime} \rrbracket\right)=\llbracket e_{1} \times e_{0}^{\prime} \rrbracket \otimes \llbracket e_{0} \times e_{1}^{\prime} \rrbracket-\llbracket e_{0} \times e_{1}^{\prime} \rrbracket \otimes \llbracket e_{1} \times e_{0}^{\prime} \rrbracket$. It follows that $\left\langle d^{1}\left(\llbracket e_{1} \times e_{1}^{\prime} \rrbracket\right), u_{1} \otimes u_{1}^{\prime}\right\rangle=1$.

4.7. Proof of Proposition 4.4. First assume $q=1$. Let $e$ be a 1-cell of $X$ and $\llbracket e \rrbracket \in H_{1}(X, \mathbb{k})$ be the homology class it represents. From the identification $I / I^{2}=\operatorname{gr}^{1}(\mathbb{k} \pi)$ and formula (5), we get $d^{1}(\llbracket e \rrbracket)=\llbracket e \rrbracket$. Thus, $d^{1}: E_{0,1}^{1} \rightarrow E_{-1,1}^{1}$ coincides with $\nabla_{X}=$ id: $H_{1}(X, \mathbb{k}) \rightarrow H_{1}(X, \mathbb{k})$. 
Now assume $q>1$. It is enough to show that $\delta^{1}\left(v_{1} \otimes v_{q-1}\right)=v_{1} \cup v_{q-1}$ over $\mathbb{k}$, for each $v_{1} \in H^{1}(X, R)$ and $v_{q-1} \in H^{q-1}(X, R)$. Let $K_{r}=K(R, r)$ be an EilenbergMacLane space as above. By obstruction theory, there is a map $f_{r}: X \rightarrow K_{r}$ such that $v_{r}=f_{r}^{*}\left(\llbracket e_{r} \rrbracket^{*}\right)$. Now set $K=K_{1} \times K_{q-1}^{\prime}$, where $K_{q-1}^{\prime}$ is a copy of $K_{q-1}$, and define $f$ to be a cellular approximation of the map $F=\left(f_{1}, f_{q-1}^{\prime}\right): X \rightarrow K$. With notation as above, $v_{1}=f^{*}\left(u_{1}\right)$ and $v_{q-1}=f^{*}\left(u_{q-1}^{\prime}\right)$, over $\mathbb{k}$. Using diagram (24) and the naturality of cup-products, the conclusion follows from Lemma 4.5 .

\section{Convergence issues}

In this section, we discuss the convergence properties of the equivariant spectral sequence $E^{\bullet}(X, M)$. Throughout this section, $\mathbb{k}$ will denote a fixed field.

5.1. The $E^{\infty}$ term. Let $C_{\bullet}=\left(C_{q}, \partial_{q}\right)$ be a chain complex over $\mathbb{k}$, endowed with an increasing filtration, $\cdots \subset F_{-2} \subset F_{-1} \subset F_{0}=C$, and let $E^{\bullet}=\left\{E_{s, t}^{r}, d^{r}\right\}_{r \geq 1}$ be the spectral sequence associated to $F_{\bullet}$. Suppose the following condition holds: For each $s$ and $t$, the $\mathbb{k}$-vector space $E_{s, t}^{1}$ is finite-dimensional. Then $E_{s, t}^{r}=E_{s, t}^{r+1}$ for all $r \geq r_{0}$. In this case, the $E^{\infty}$ term is defined as $E_{s, t}^{\infty}=E_{s, t}^{r_{0}}$, and the inclusion

$$
E_{s, t}^{\infty} \supseteq \operatorname{gr}^{s}\left(H_{s+t}(C)\right)
$$

holds for all $s, t$.

The spectral sequence is said to be convergent if $E_{s, t}^{\infty}=\operatorname{gr}^{s}\left(H_{s+t}(C)\right)$, for all $s, t$. For short, we write $E_{s, t}^{1} \Rightarrow H_{s+t}(C)$. Recall $Z_{s}^{r}=\left\{z \in F_{s} \mid \partial z \in F_{s-r}\right\}$, and write $Z_{s}^{\infty}=\left\{z \in F_{s} \mid \partial z=0\right\}$. In this setup, convergence of $E^{\bullet}$ is equivalent to

$$
\bigcap_{r}\left(Z_{s}^{r}+F_{s-1}\right) \subseteq Z_{s}^{\infty}+F_{s-1}, \quad \text { for all } s .
$$

5.2. The $E^{\infty}$ term of the equivariant spectral sequence. Now let $X$ be a connected CW-complex, with fundamental group $\pi=\pi_{1}(X)$. Let $I=I_{\mathbb{k}}(\mathbb{k} \pi)$ be the augmentation ideal of $\mathbb{k} \pi$, and let $M$ be a right $\mathbb{k} \pi$-module. Under fairly general assumptions, the $I$-adic spectral sequence $E^{\bullet}(X, M)$ has an $E^{\infty}$ term. Its convergence, though, is a rather delicate matter.

Proposition 5.1. Suppose $\operatorname{dim}_{\mathbb{k}} H_{q}(X, \mathbb{k})<\infty$, for all $q \geq 0$, and $\operatorname{dim}_{\mathbb{k}} M / M I<$ $\infty$. Then, the spectral sequence $E^{\bullet}(X, M)$ has an $E^{\infty}$ term.

Proof. Recall that $\operatorname{gr}(M)$ is generated as a $\operatorname{gr}(\mathbb{k} \pi)$-module by $\operatorname{gr}^{0}(M)=M / M I$ and $\operatorname{gr}(\mathbb{k} \pi)$ is generated as a ring by $\operatorname{gr}^{1}(\mathbb{k} \pi)=H_{1}(X, \mathbb{k})$. We infer that $\operatorname{dim}_{\mathbb{k}} \operatorname{gr}^{s}(M)<$ $\infty$, for all $s$. Hence, for any fixed $s$ and $t$, the vector space $E_{-s, t}^{1}=\operatorname{gr}^{s}(M) \otimes_{\mathrm{k}}$ $H_{t-s}(X, \mathbb{k})$ is finite-dimensional. The conclusion follows.

Remark 5.2. Let $X$ be a finite-type CW-complex, and suppose $M=\mathbb{k} \pi$. Then $M / M I=\mathbb{k}$, and so the $E^{\infty}$ term exists. More generally, suppose $\nu: \pi \rightarrow G$ is an epimorphism. Take $M=\mathbb{k} G_{\nu}$, and let $J=I_{\mathbb{k}} G$. Then $M / M I=\mathbb{k} G / J=\mathbb{k}$, and so $E^{\infty}$ exists.

5.3. A non-convergent spectral sequence. We now give an example of a CWcomplex $X$ and a $\mathbb{k} \pi$-module $M$ for which the assumptions of Proposition 5.1 are satisfied, yet the spectral sequence $E^{\bullet}(X, M)$ does not converge. More precisely, for each integer $m \geq 3$, we construct a finite CW-complex $X$ of dimension $m$ for which the convergence condition (28) fails for the coefficient module $M=\mathbb{k} \pi$, in filtration degree $s=0$ and total degree $m$. 


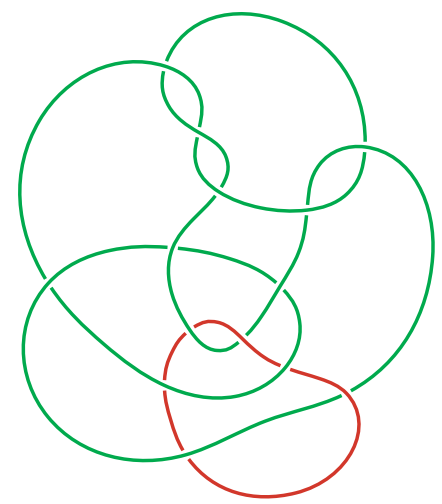

FiguRE 1. Hillman's 2-component link

Example 5.3. Let $L$ be the 2-component link in $S^{3}$ depicted in Figure 11 and let $\pi=\pi_{1}\left(S^{3} \backslash L\right)$ be the fundamental group of its complement. In [16, J. Hillman showed that this group is not residually nilpotent; that is, if $\left\{\Gamma_{n}(\pi)\right\}_{n \geq 1}$ is the lower central series of $\pi$, then $\Gamma_{\omega}(\pi):=\bigcap_{n \geq 1} \Gamma_{n}(\pi)$ is non-trivial. Pick an element $1 \neq g \in \Gamma_{\omega}(\pi)$, and set $x=g-1 \in I$. From [26], we know that the map $\pi \hookrightarrow \mathbb{k} \pi$, $h \mapsto h-1$, sends $\Gamma_{n}(\pi)$ to $I^{n}$, for all $n \geq 1$. Hence, $x$ belongs to the $I$-adic radical, $I^{\omega}:=\bigcap_{n \geq 1} I^{n}$.

The link complement has the homotopy type of a finite 2-complex, say $X_{0}$. Fix an integer $m \geq 3$. Using the construction from Example 2.1. we may define a CW-complex

$$
X=\left(X_{0} \vee S^{m-1}\right) \cup_{\phi_{x}} e_{m},
$$

with $\left[\phi_{x}\right]=x \cdot\left[S^{m-1}\right] \in \pi_{m-1}\left(X_{0} \vee S^{m-1}\right)$. Clearly, $\pi_{1}(X)=\pi$. We know that $C_{\bullet}(\widetilde{X}, \mathbb{k})=C \bullet\left(\widetilde{X}_{0}, \mathbb{k}\right) \oplus C(m-1, x)$, and the differential of $C(m-1, x)$, $\tilde{\partial}_{m}: \mathbb{k} \pi \rightarrow \mathbb{k} \pi$, sends $1 \in \mathbb{k} \pi$ to $x \in \mathbb{k} \pi$.

From the definitions, $Z_{0, m}^{r}=\left\{z \in \mathbb{k} \pi \mid \tilde{\partial}_{m}(z) \in I^{r}\right\}$; hence, $1 \in \bigcap_{r} Z_{0, m}^{r}$. On the other hand, $Z_{0, m}^{\infty}=\operatorname{ker}\left(\tilde{\partial}_{m}\right)$. Thus, if (28) were to hold, we would have

$$
1 \in \operatorname{ker}\left(\tilde{\partial}_{m}\right)+I .
$$

Now, $\operatorname{ker}\left(\tilde{\partial}_{m}\right)=\{y \in \mathbb{k} \pi \mid y x=0\}$, and this subspace vanishes. Indeed, every link group is locally indicable, by [18], and the group algebra of a locally indicable group has no zero divisors, by [15]. We conclude that $1 \in I$, a contradiction.

\section{BASE ChANGe AND FiLtrations ON HOMOLOGY}

We now turn to the special case when the module $M$ is actually a ring $R$, with the right $\mathbb{k} \pi$-module structure given by an extension of scalars. We analyze the $d^{1}$ differential of $E^{1}(X, R)$, and two natural filtrations on $H_{*}(X, R)$.

\footnotetext{
${ }^{1}$ This picture was drawn with the help of the Mathematica package KnotTheory, by Dror Bar-Natan, and the graphical package Knotilus, by Ortho Flint and Stuart Rankin.
} 
6.1. Base change. Let $\pi$ be a group and $\mathbb{k}$ a commutative, unital ring. Suppose we are given a ring $R$ and a ring homomorphism $\rho: \mathbb{k} \pi \rightarrow R$ (also known as a 'base change' or 'extension of scalars'). Then $R$ becomes a right $\mathbb{k} \pi$-module by setting $r \cdot x=r \rho(x)$ for all $x \in \mathbb{k} \pi$ and $r \in R$. We will denote by $J$ the two-sided ideal of $R$ generated by $\rho(I)$, where $I$ is the augmentation ideal of $\mathbb{k} \pi$.

A particular case arises when we are given a group homomorphism, $\nu: \pi \rightarrow G$. Then, the linear extension of $\nu$ to group rings, call it $\bar{\nu}: \mathbb{k} \pi \rightarrow \mathbb{k} G$, is a ring homomorphism. As in Example 2.3, we will denote the resulting $\mathbb{k} \pi$-module by $\mathbb{k} G_{\nu}$.

Lemma 6.1. With notation as above, suppose $R$ is a commutative ring or $R=\mathbb{k} G_{\nu}$ for some epimorphism $\nu: \pi \rightarrow G$. Then:

(1) $R I^{n}=J^{n}$, for all $n \geq 0$. If $R=\mathbb{k} G_{\nu}$, then $J=I_{\mathbb{k}} G$.

(2) $\operatorname{gr}_{I}(R)=\operatorname{gr}_{J}(R)$.

Proof. (11) By definition, $J=R \rho(I) R$, and so the inclusion $R I^{n} \subset J^{n}$ always holds. If $R$ is commutative, the reverse inclusion is obvious.

Now suppose $R=\mathbb{k} G_{\nu}$. Recall $I=I_{\mathbb{k}} \pi$ is generated (as a $\mathbb{k}$-module) by all elements of the form $g-1$, with $g \in \pi$. Since $\nu$ is surjective, it follows that $\bar{\nu}(I)=I_{\mathrm{k}} G$. In particular, $\bar{\nu}(I)$ is a two-sided ideal of $R$, so $J=I_{\mathrm{k}} G$ and $J^{n} \subset R I^{n}$.

(2) Follows from (11).

6.2. The spectral sequence with $R$-coefficients. Let $X$ be a connected CWcomplex with a single 0 -cell $e_{0}$ and fundamental group $\pi=\pi_{1}\left(X, e_{0}\right)$. Suppose $R$ is a ring, endowed with the right $\mathbb{k} \pi$-module structure given by a ring homomorphism $\rho: \mathbb{k} \pi \rightarrow R$. Then the $I$-adic spectral sequence $E^{\bullet}(X, R)$ is a spectral sequence in the category of left $R$-modules. Indeed, $R$ acts on itself by left-multiplication, and this action extends in a natural way to each term $E^{r}(X, R)$. Furthermore, all differentials $d_{R}^{r}: E^{r} \rightarrow E^{r}$ are $R$-linear.

Now assume $R$ satisfies one of the two conditions of Lemma 6.1. As above, let $J$ be the two-sided ideal of $R$ generated by $\rho(I)$. Consider the $R$-chain complex $C_{\bullet}(X, R)=R \otimes_{\mathbb{k}} C_{\bullet}(X, \mathbb{k})$, with a differential $\tilde{\partial}^{R}=\operatorname{id}_{R} \otimes_{\mathbb{k} \pi} \tilde{\partial}$ and a filtration $F^{\bullet}$ given by (11). By Lemma 6.1, the terms of this filtration can be expressed as

$$
F^{n} C_{\bullet}(X, R)=J^{n} \otimes_{\mathbb{k}} C_{\bullet}(X, \mathbb{k}),
$$

for all $n \geq 0$. Hence, by (15),

$$
E_{-s, t}^{1}(X, R)=H_{t-s}\left(X, \operatorname{gr}_{J}^{s}(R)\right) .
$$

Lemma 6.2. Suppose $R$ is a commutative ring, or $R=\mathbb{k} G_{\nu}$, for some epimorphism $\nu: \pi \rightarrow G$. Then $E^{\bullet}(X, R)$ is a spectral sequence in the category of left $\operatorname{gr}_{J}(R)$ modules.

Proof. Let $Z_{-s}^{r}=\left\{z \in F_{-s} \mid \tilde{\partial}^{R} z \in F_{-s-r}\right\}$. By construction, the terms of the spectral sequence are given by

$$
E_{-s}^{r}=Z_{-s}^{r} /\left(Z_{-(s+1)}^{r-1}+\tilde{\partial}^{R} Z_{-(s+1-r)}^{r-1}\right) .
$$

From (29), we find that $J^{n} \cdot Z_{-s}^{r} \subset Z_{-s-n}^{r}$, for all $n \geq 0$. Using these inclusions, together with (31), we infer that

$$
J^{n} \cdot E_{-s}^{r} \subset E_{-s-n}^{r}, \quad \text { for all } n \geq 0 .
$$


This allows us to define a natural gr ${ }_{J}(R)$-module structure on ${ }_{q} E^{r}=\bigoplus_{s \geq 0} E_{-s, s+q}^{r}$, for all $q \geq 0$, and thus on $E^{r}=\bigoplus_{q \geq 0}{ } E^{r}$, for all $r \geq 1$. (The $\operatorname{gr}_{J}(R)$-module structure on ${ }_{q} E^{1}=\bigoplus_{s \geq 0} H_{q}\left(X, \operatorname{gr}_{J}^{s}(R)\right)$ is induced by left-multiplication.)

Using (31) once again, it is readily checked that the differentials $d_{R}^{r}:{ }_{q} E^{r} \rightarrow$ ${ }_{q-1} E^{r} \operatorname{act}_{\operatorname{gr}_{J}}(R)$-linearly, for all $r \geq 1$.

6.3. The differential of $E^{1}\left(X, \mathbb{k} G_{\nu}\right)$. In this subsection, we assume that either $\mathbb{k}=\mathbb{Z}$ and $H_{*}(X, \mathbb{Z})$ is torsion-free, or $\mathbb{k}$ is a field. In this case, ${ }_{q} E^{1}=\operatorname{gr}_{J}(R) \otimes_{\mathbb{k}}$ $H_{q}(X, \mathbb{k})$, by (20); in particular, ${ }_{q} E^{1}$ is a free $\operatorname{gr}_{J}(R)$-module, for all $q \geq 0$.

The above lemma tells us that, in order to describe $d_{R}^{1}$ completely, it is enough to identify its effect on free $\operatorname{gr}_{J}(R)$-module generators. We will do this now, in the case when the ring $R$ is a group-ring, $\mathbb{k} G$, with $\mathbb{k} \pi$-module structure given by $\bar{\nu}: \mathbb{k} \pi \rightarrow \mathbb{k} G$, the linear extension of an epimorphism $\nu: \pi \rightarrow G$.

As above, let $J=I_{\mathbb{k}} G$ be the two-sided ideal of $\mathbb{k} G$ generated by $\bar{\nu}(I)$, where $I=I_{\mathbb{k}} \pi$. Let

$$
\nu_{*}: H_{1}(X, \mathbb{k}) \longrightarrow H_{1}(G, \mathbb{k})
$$

be the homomorphism induced by $\nu$ in homology with coefficients in $\mathbb{k}$. In view of Lemma 6.2, the next result describes the differential $d_{G}^{1}$ of $E^{1}\left(X, \mathbb{k} G_{\nu}\right)$, solely in terms of $\nu_{*}$ and the comultiplication map $\nabla_{X}$ in $H_{*}(X, \mathbb{k})$.

Proposition 6.3. The restriction of $d_{G}^{1}$ to $1 \otimes H_{q}(X, \mathbb{k})$ is the composite

$$
H_{q}(X, \mathbb{k}) \stackrel{\nabla_{X}}{\longrightarrow} H_{1}(X, \mathbb{k}) \otimes_{\mathbb{k}} H_{q-1}(X, \mathbb{k}) \stackrel{\nu_{*} \otimes \mathrm{id}}{\longrightarrow} H_{1}(G, \mathbb{k}) \otimes_{\mathbb{k}} H_{q-1}(X, \mathbb{k}) .
$$

Proof. Let $\mu: \mathbb{k} G \otimes_{\mathbb{k}} \mathbb{k} \pi \rightarrow \mathbb{k} G$ be the multiplication map of the $\mathbb{k} \pi$-module $\mathbb{k} G$, given on group elements by $\mu(g \otimes x)=g \nu(x)$. In view of Theorem 4.1, we only need to identify the map

$$
\operatorname{gr}(\mu): \operatorname{gr}_{J}^{0}(\mathbb{k} G) \otimes_{\mathbb{k}} \operatorname{gr}_{I}^{1}(\mathbb{k} \pi) \rightarrow \operatorname{gr}_{J}^{1}(\mathbb{k} G)
$$

Under the identification $\operatorname{gr}_{J}^{0}(\mathbb{k} G)=\mathbb{k}$, this map coincides with $\operatorname{gr}^{1}(\bar{\nu}): \operatorname{gr}_{I}^{1}(\mathbb{k} \pi) \rightarrow$ $\operatorname{gr}_{J}^{1}(\mathbb{k} G)$. In turn, under the identifications $\operatorname{gr}_{I}^{1}(\mathbb{k} \pi)=H_{1}(X, \mathbb{k})$ and $\operatorname{gr}_{J}^{1}(\mathbb{k} G)=$ $H_{1}(G, \mathbb{k})$ provided by (2), the map $\operatorname{gr}^{1}(\bar{\nu})$ coincides with $\nu_{*}$. This finishes the proof.

The next corollary describes (under some mild hypothesis) the dual of the differential $d_{G}^{1}$, solely in terms of the cup-product structure on $H^{*}(X, \mathbb{k})$, and the homomorphism $\nu^{*}: H^{1}(G, \mathbb{k}) \hookrightarrow H^{1}(X, \mathbb{k})$ induced in cohomology by the epimorphism $\nu: \pi \rightarrow G$.

Corollary 6.4. Suppose $X$ is a finite-type $C W$-complex, and $H_{1}(G, \mathbb{k})$ is a free $\mathbb{k}$-module. Let $\delta_{G}^{1}$ be the transpose of the restriction of $d_{G}^{1}$ to $1 \otimes H_{q}(X, \mathbb{k})$. We then have a commuting triangle

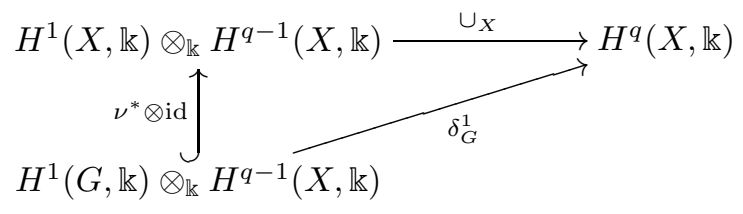


6.4. Filtrations on homology. Let $X$ be a connected CW-complex as before, let $\mathbb{k}$ be a commutative ring, and let $M$ be a right $\mathbb{k} \pi$-module. The $I$-adic filtration on $C \cdot(X, M)$ naturally defines a descending filtration on $H_{*}(X, M)$. The $n$-th term of the filtration is given by

$$
F^{n} H_{r}(X, M)=\operatorname{im}\left(H_{r}\left(F^{n} C_{\bullet}(X, M)\right) \longrightarrow H_{r}(X, M)\right) .
$$

Clearly, $F^{0} H_{r}(X, M)=H_{r}(X, M)$. As usual, we will write $F_{-n}=F^{n}$. The associated graded object of this filtration will be denoted by $\operatorname{gr}_{F}\left(H_{*}(X, M)\right)$.

The filtration (32) need not be separated, even when the spectral sequence converges. We shall illustrate this phenomenon in Example 8.5.

Now consider the case when $M$ is a ring $R$, with right $\mathbb{k} \pi$-module structure given by a base change $\rho: \mathbb{k} \pi \rightarrow R$. Let $J$ be the two-sided ideal generated by $\rho(I)$. As before, we will assume that either $R$ is commutative or $R=\mathbb{k} G$, and $\rho=\bar{\nu}$, for some epimorphism $\nu: \pi \rightarrow G$.

Using (29), we find that the spectral sequence filtration on $H_{*}(X, R)$, as defined in (32), is given by

$$
F^{n} H_{*}(X, R)=\left(\operatorname{ker} \tilde{\partial}^{R} \cap J^{n} C_{\bullet}(X, R)+\operatorname{im} \tilde{\partial}^{R}\right) / \operatorname{im} \tilde{\partial}^{R} .
$$

This puts a natural $\operatorname{gr}_{J}(R)$-module structure on $\operatorname{gr}_{F} H_{*}(X, R)$, since plainly

$$
J^{k} \cdot\left(J^{n} C_{\bullet}(X, R) \cap \operatorname{ker} \tilde{\partial}^{R}\right) \subset J^{k+n} C_{\bullet}(X, R) \cap \operatorname{ker} \tilde{\partial}^{R}, \quad \forall k, n .
$$

Remark 6.5. Suppose $X$ is of finite type, $\mathbb{k}$ is a field, and $\operatorname{dim}_{\mathbb{k}} R / J<\infty$ (this happens automatically when $R=\mathbb{k} G_{\nu}$, in which case $R / J=\mathbb{k}$ ). Fix an integer $q \geq 0$. Applying Proposition 5.1 and making use of (27), we obtain an inclusion

$$
\operatorname{gr}_{F} H_{q}(X, R) \subset{ }_{q} E^{\infty}=\bigoplus_{s \geq 0} E_{-s, s+q}^{\infty},
$$

which is compatible with the respective $\operatorname{gr}_{J}(R)$-actions.

6.5. Comparing the two filtrations. We now have two filtrations on $H_{q}(X, R)$ : the spectral sequence filtration $F^{\bullet} H_{q}(X, R)$, given by (33), and the $J$-adic filtration, $J^{\bullet} \cdot H_{q}(X, R)$. The two filtrations are related as follows.

Lemma 6.6. $J^{k} \cdot F^{n} H_{*}(X, R) \subseteq F^{k+n} H_{*}(X, R)$, for all $k, n \geq 0$.

Proof. Our claim follows from (33) and (34).

Corollary 6.7. The $J$-adic filtration on $H_{*}(X, R)$ is finer than the filtration defined by $I$-adic spectral sequence:

$$
J^{k} \cdot H_{*}(X, R) \subseteq F^{k} H_{*}(X, R), \quad \text { for all } k \geq 0 .
$$

In general, the two filtrations differ, even in the case when $R=\mathbb{k} A$, with $A$ an abelian group, as the next example shows. On the other hand, we will see in Lemma 8.4 that the two filtrations coincide for $A=\mathbb{Z}$.

Example 6.8. Consider the space $X=S^{1} \vee S^{1}$ and its universal abelian cover, $X^{\mathrm{ab}} \rightarrow X$, classified by the map ab: $\pi_{1}(X) \rightarrow \mathbb{Z}^{2}$. The chain complex $C_{\bullet}\left(X^{\mathrm{ab}}, \mathbb{k}\right)$ takes the form $\tilde{\partial}_{1}: \Lambda^{2} \rightarrow \Lambda$, where $\Lambda=\mathbb{k}\left[t_{1}^{ \pm 1}, t_{2}^{ \pm 1}\right]$ and $\tilde{\partial}_{1}=\left(\begin{array}{c}t_{1}-1 \\ t_{2}-1\end{array}\right)$. Hence,

$$
H_{1}\left(X^{\mathrm{ab}}, \mathbb{k}\right)=\operatorname{ker} \tilde{\partial}_{1}=\operatorname{im}\left(t_{2}-1,1-t_{1}\right) \cong \Lambda \text {. }
$$

It follows that $J^{s} \cdot H_{1}\left(X^{\mathrm{ab}}, \mathbb{k}\right) \cong J^{s}$. On the other hand, $F_{-s} H_{1}\left(X^{\mathrm{ab}}, \mathbb{k}\right) \cong J^{s-1}$. Thus, the two filtrations on $H_{1}\left(X^{\mathrm{ab}}, \mathbb{k}\right)$ do not coincide. 


\section{The homological Reznikov spectral Sequence}

In this section, we specialize in the case where the coefficient module is the groupring $\mathbb{k} G$ of a cyclic group of prime-power order $p^{r}$ over a field $\mathbb{k}$ of characteristic $p$. When $X=K(\pi, 1)$ is an Eilenberg-MacLane space and $G=\mathbb{Z}_{p}$, the resulting spectral sequence is the homological version of a cohomology spectral sequence described by Reznikov in [27].

7.1. A convergent spectral sequence. Let $X$ be a connected $\mathrm{CW}$-complex, and let $\pi=\pi_{1}(X)$. Assume we have an epimorphism $\nu: \pi \rightarrow \mathbb{Z}_{p^{r}}$. Fix a field $\mathbb{k}$ of characteristic $p$, and denote by $\left(\mathbb{k}_{p^{r}}\right)_{\nu}$ the group-ring of $\mathbb{Z}_{p^{r}}$, viewed as a right $\mathbb{k} \pi$-module via the linear extension $\bar{\nu}: \mathbb{k} \pi \rightarrow \mathbb{k}_{p^{r}}$. Let $I=I_{\mathbb{k}}(\pi)$ and $J=I_{\mathbb{k}}\left(\mathbb{Z}_{p^{r}}\right)$ be the respective augmentation ideals.

Lemma 7.1. The I-adic filtration on $C \bullet\left(X,\left(\mathbb{k}_{p^{r}}\right)_{\nu}\right)$ is finite.

Proof. By (29), $F^{n} C_{\bullet}\left(X,\left(\mathbb{k}_{p^{r}}\right)_{\nu}\right)=J^{n} \otimes_{\mathbb{k}} C_{\bullet}(X, \mathbb{k})$, for all $n \geq 0$. Now identify $\mathbb{k} \mathbb{Z}_{p^{r}}=\mathbb{k}[t] /\left(t^{p^{r}}-1\right)$, and note that $J$ is the ideal generated by $(t-1)$. By the binomial formula, $(t-1)^{p^{r}}=t^{p^{r}}-1$ over $\mathbb{k}$. Thus, $J^{p^{r}}=0$. Putting things together, we conclude that $F^{p^{r}} C \bullet\left(X,\left(\mathbb{k}_{p^{r}}\right)_{\nu}\right)=0$.

Therefore, $E^{\bullet}\left(X,\left(\mathbb{k} \mathbb{Z}_{p^{r}}\right)_{\nu}\right)$ converges. In fact, the spectral sequence collapses in finitely many steps: $\bigoplus_{s+t=q} E_{s, t}^{p^{r}}\left(X,\left(\mathbb{k} \mathbb{Z}_{p^{r}}\right)_{\nu}\right)=H_{q}\left(X,\left(\mathbb{k} \mathbb{Z}_{p^{r}}\right)_{\nu}\right)$. For another situation when the spectral sequence converges, see Proposition 8.1 below.

Nevertheless, the spectral sequence filtration, $F^{\bullet} H_{*}\left(X,\left(\mathbb{k} \mathbb{Z}_{p^{r}}\right)_{\nu}\right)$, does not always coincide with the $J$-adic filtration, $J^{\bullet} \cdot H_{*}\left(X,\left(\mathbb{k}_{p^{r}}\right)_{\nu}\right)$, as the following example shows.

Example 7.2. Let $X=S^{1}$, and consider the canonical projection $\nu: \pi_{1}\left(S^{1}\right)=\mathbb{Z} \rightarrow$ $\mathbb{Z}_{p}$. Identifying $\mathbb{F}_{p} \mathbb{Z}_{p}$ with $\Lambda_{p}=\mathbb{F}_{p}[t] /\left(t^{p}-1\right)$, the chain complex $C_{\bullet}\left(S^{1},\left(\mathbb{F}_{p} \mathbb{Z}_{p}\right)_{\nu}\right)$ takes the form $\tilde{\partial}_{1}: \Lambda_{p} \rightarrow \Lambda_{p}$, with $\tilde{\partial}_{1}=(t-1)$. It follows that $H_{1}\left(S^{1}, \Lambda_{p}\right)=$ $\operatorname{ker}\left(\tilde{\partial}_{1}\right)$ is the ideal generated by the norm element, $N=1+\cdots+t^{p-1}$. Hence, $J \cdot H_{1}\left(S^{1}, \Lambda_{p}\right)=0$.

On the other hand, $N$ augments to 0 in $\mathbb{F}_{p}$, and so $N \in J \cap \operatorname{ker}\left(\tilde{\partial}_{1}\right)=F^{1} H_{1}\left(S^{1}, \Lambda_{p}\right)$. Thus, $J \cdot H_{1}\left(S^{1}, \Lambda_{p}\right) \neq F^{1} H_{1}\left(S^{1}, \Lambda_{p}\right)$.

7.2. The Reznikov spectral sequence. We now specialize to the case when $G=$ $\mathbb{Z}_{p}$ and $\mathbb{k}=\mathbb{F}_{p}$. Identify $\mathbb{F}_{p} \mathbb{Z}_{p}$ with $\mathbb{F}_{p}[t] /\left(t^{p}-1\right)$ and $\operatorname{gr}_{J}\left(\mathbb{F}_{p} \mathbb{Z}_{p}\right)$ with $\mathbb{F}_{p}[x] /\left(x^{p}\right)$, where $x=t-1$. Let $\nu: \pi \rightarrow \mathbb{Z}_{p}$ be an epimorphism, and consider the spectral sequence $E^{\bullet}\left(X,\left(\mathbb{F}_{p} \mathbb{Z}_{p}\right)_{\nu}\right)$. By $(\underline{30})$, the first term is

$$
E_{-s, t}^{1}=\operatorname{gr}_{J}^{s}\left(\mathbb{F}_{p} \mathbb{Z}_{p}\right) \otimes_{\mathbb{F}_{p}} H_{t-s}\left(X, \mathbb{F}_{p}\right)=H_{t-s}\left(X, \mathbb{F}_{p}\right)
$$

for $t-s \geq 0$ and $0 \leq s \leq p-1$, and 0 otherwise. Here we used $\operatorname{gr}_{J}^{s}\left(\mathbb{F}_{p} \mathbb{Z}_{p}\right)=\mathbb{F}_{p}$ with a basis $x^{s}$ in the specified range.

To identify the $d^{1}$ differential, let $\nu_{*}: H_{1}\left(X, \mathbb{F}_{p}\right) \rightarrow H_{1}\left(\mathbb{Z}_{p}, \mathbb{F}_{p}\right)=\mathbb{F}_{p}$ be the homomorphism induced by $\nu$ in homology, and let $\nu_{\mathbb{F}_{p}} \in H^{1}\left(X, \mathbb{F}_{p}\right)$ be the corresponding cohomology class.

Proposition 7.3. Under the identification from (36), the differential $d^{1}: E_{-s, s+q}^{1}$ $\rightarrow E_{-s-1, s+q}^{1}$ is the composite

$$
\varrho_{q}: H_{q}\left(X, \mathbb{F}_{p}\right) \stackrel{\nabla_{X}}{\longrightarrow} H_{1}\left(X, \mathbb{F}_{p}\right) \otimes_{\mathbb{F}_{p}} H_{q-1}\left(X, \mathbb{F}_{p}\right) \stackrel{\nu_{*} \otimes \mathrm{id}}{\longrightarrow} \mathbb{F}_{p} \otimes_{\mathbb{F}_{p}} H_{q-1}\left(X, \mathbb{F}_{p}\right)
$$


If, moreover, $X$ is of finite type, then the dual $\delta^{1}=\left(d^{1}\right)^{*}$ coincides with the leftmultiplication map

$$
\cdot \nu_{\mathbb{F}_{p}}: H^{q-1}\left(X, \mathbb{F}_{p}\right) \rightarrow H^{q}\left(X, \mathbb{F}_{p}\right) .
$$

Proof. For the first statement, use Proposition 6.3 with $G=\mathbb{Z}_{p}$ and $\mathbb{k}=\mathbb{F}_{p}$, noting that under the identifications $\mathbb{F}_{p} \cong H_{1}\left(\mathbb{Z}_{p}, \mathbb{F}_{p}\right) \cong J / J^{2} \cong \mathbb{F}_{p} \cdot x$, the unit 1 corresponds to $x$. The second statement follows from Corollary 6.4.

In the particular case when $X=K(\pi, 1)$, we recover the spectral sequence from [27, Theorem 13.1] in homological form.

Corollary 7.4. Let $1 \rightarrow K \rightarrow \pi \stackrel{\nu}{\rightarrow} \mathbb{Z}_{p} \rightarrow 1$ be an exact sequence of groups. There is then a spectral sequence

$$
E_{-s, s+q}^{1}=H_{q}\left(\pi, \mathbb{F}_{p}\right) \Rightarrow H_{q}\left(K, \mathbb{F}_{p}\right),
$$

$q \geq 0$ and $0 \leq s \leq p-1$, with differential $d^{1}: H_{q}\left(\pi, \mathbb{F}_{p}\right) \rightarrow H_{q-1}\left(\pi, \mathbb{F}_{p}\right)$ equal to $\varrho_{q}=\left(\nu_{*} \otimes \mathrm{id}\right) \circ \nabla_{K(\pi, 1)}$, and converging in finitely many steps.

7.3. Monodromy action. In view of Proposition 7.3, the first page of the spectral sequence $E^{\bullet}\left(X,\left(\mathbb{F}_{p} \mathbb{Z}_{p}\right)_{\nu}\right)$ looks like

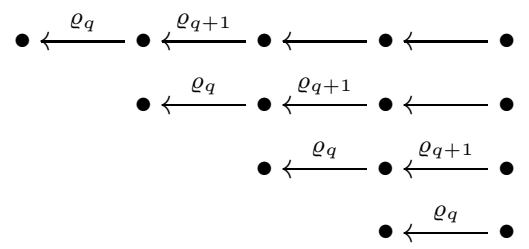

with columns running right to left from $E_{0, *}^{1}$ to $E_{-p+1, *}^{1}$. Thus, if $p \neq 2$, we have a chain complex,

$$
\cdots \longrightarrow H_{q+1}\left(X, \mathbb{F}_{p}\right) \stackrel{\varrho_{q+1}}{\longrightarrow} H_{q}\left(X, \mathbb{F}_{p}\right) \stackrel{\varrho_{q}}{\longrightarrow} H_{q-1}\left(X, \mathbb{F}_{p}\right) \longrightarrow \cdots .
$$

Proposition 7.5. If the chain complex $\left(H_{*}\left(X, \mathbb{F}_{p}\right), \varrho_{*}\right)$ is acyclic in degree $*=q$, then $J^{2} \cdot H_{q}\left(X,\left(\mathbb{F}_{p} \mathbb{Z}_{p}\right)_{\nu}\right)=0$.

Proof. Let $F^{n}=F^{n} H_{q}\left(X,\left(\mathbb{F}_{p} \mathbb{Z}_{p}\right)_{\nu}\right)$ be the spectral sequence filtration. Our hypothesis forces $E_{-s, q+s}^{2}=0$, for $0<s<p-1$. Hence, $E_{-s, q+s}^{\infty}=0$, for $0<s<p-1$, which implies $F^{1}=\cdots=F^{p-1}$.

Now recall from Lemma 6.6 that $J^{k} \cdot F^{n} \subseteq F^{k+n}$. Hence

$$
J^{2} \cdot H_{q}\left(X,\left(\mathbb{F}_{p} \mathbb{Z}_{p}\right)_{\nu}\right)=J^{2} F^{0} \subseteq J F^{1}=J F^{p-1} \subseteq F^{p}=0,
$$

and this finishes the proof.

\section{Completion And homology of abelian COVERS}

In this section, we study the more general situation when the coefficient module is the group-ring of an abelian group $A$, with emphasis on the good convergence properties of the corresponding equivariant spectral sequence. In the process, we use standard completion tools from commutative algebra, as described in 20] and [30. 
8.1. Convergence. Let $\mathbb{k}$ be a field. Suppose we are given an epimorphism $\nu: \pi \rightarrow$ $A$ onto an abelian group. We may then view the ring $\Lambda:=\mathbb{k} A$ as a right $\mathbb{k} \pi$-module, via the ring map $\bar{\nu}: \mathbb{k} \pi \rightarrow \mathbb{k} A$. We shall denote this module by $\Lambda_{\nu}$ and will consider the spectral sequence $E^{\bullet}\left(X, \Lambda_{\nu}\right)$ associated to the Galois cover of the CW-complex $X$ corresponding to $\nu$.

As usual, write $I=I_{\mathbb{k}} \pi$ and $J=\bar{\nu}(I) \Lambda$, and recall that $J$ is the augmentation ideal of $\Lambda=\mathbb{k} A$. Also, note that $J$ is a maximal ideal of the Noetherian ring $\Lambda$. Let $\iota: \Lambda \rightarrow \widehat{\Lambda}$ be the $J$-adic completion. Set $\widehat{J}:=\iota(J) \widehat{\Lambda}$, and note that $\widehat{\Lambda}$ is a Noetherian local ring with maximal ideal $\widehat{J}$, hence a Zariski ring. The ring morphism $\hat{\nu}:=\iota \circ \bar{\nu}$ defines another $\mathbb{k} \pi$-module, which we will denote by $\widehat{\Lambda}_{\hat{\nu}}$. We thus obtain a morphism of spectral sequences,

$$
E^{\bullet}\left(X, \Lambda_{\nu}\right) \stackrel{E^{\bullet}(\iota)}{\longrightarrow} E^{\bullet}\left(X, \widehat{\Lambda}_{\hat{\nu}}\right) .
$$

We will frequently make use of the fact that, for any finitely generated $\Lambda$-module $M$, there is a natural $\widehat{\Lambda}$-isomorphism,

$$
M \otimes_{\Lambda} \widehat{\Lambda} \stackrel{\cong}{\longrightarrow} \widehat{M}
$$

and the completion filtration on the $J$-adic completion $\widehat{M}$ coincides with the $\widehat{J}$-adic filtration.

The good convergence properties of both spectral sequences follow easily from the Artin-Rees Lemma, as explained by Serre in [30].

Proposition 8.1. Let $X$ be a connected $C W$-complex of finite type. With notation as above, we have:

(1) $E_{s, t}^{1}\left(X, \Lambda_{\nu}\right) \Rightarrow H_{s+t}\left(X, \Lambda_{\nu}\right)$ and $E_{s, t}^{1}\left(X, \widehat{\Lambda}_{\hat{\nu}}\right) \Rightarrow H_{s+t}\left(X, \widehat{\Lambda}_{\hat{\nu}}\right)$.

(2) The filtration $F_{\bullet} H_{*}\left(X, \widehat{\Lambda}_{\hat{\nu}}\right)$ is separated.

(3) If $X$ is a finite complex, the spectral sequence $E^{\bullet}\left(X, \Lambda_{\nu}\right)$ collapses in finitely many steps.

Proof. See [30, pp. 22-24].

The next lemma shows that $E^{\bullet}\left(X, \Lambda_{\nu}\right)$ computes $H_{*}\left(X, \Lambda_{\nu}\right) \otimes_{\Lambda} \widehat{\Lambda}$, the $J$-adic completion of $H_{*}\left(X, \Lambda_{\nu}\right)$.

Lemma 8.2. Let $X$ be a connected $C W$-complex of finite type. Then the maps

$$
E^{r}(\iota): E^{r}\left(X, \Lambda_{\nu}\right) \rightarrow E^{r}\left(X, \widehat{\Lambda}_{\hat{\nu}}\right) \quad \text { and } \quad \operatorname{gr}_{F}(\iota): \operatorname{gr}_{F} H_{q}\left(X, \Lambda_{\nu}\right) \rightarrow \operatorname{gr}_{F} H_{q}\left(X, \widehat{\Lambda}_{\hat{\nu}}\right)
$$

are isomorphisms, for all $1 \leq r \leq \infty$ and $q \geq 0$.

Proof. Set $C_{\bullet}:=C_{\bullet}(X, \Lambda)$ and $\widehat{C}_{\bullet}:=C_{\bullet}(X, \widehat{\Lambda})$. Note that, by construction, $\widehat{C}_{\bullet}$ is the $J$-adic completion of the $\Lambda$-chain complex $C_{\bullet}$. By (29),

$$
F^{k} C_{\bullet}(X, \Lambda)=J^{k} \cdot C_{\bullet} \text { and } F^{k} C_{\bullet}(X, \widehat{\Lambda})=\widehat{J}^{k} \cdot \widehat{C}_{\bullet}
$$

for all $k$. Since $\iota: C_{\bullet} \rightarrow \widehat{C}_{\bullet}$ is the completion map, it follows from (37) that $E^{0}(\iota)$ is an isomorphism. Therefore, $E^{r}(\iota)$ is an isomorphism, for $1 \leq r<\infty$. The remaining assertions follow from convergence. 
8.2. Cyclic quotients. We now specialize further, to the case when $A=\mathbb{Z}$. Let $\nu: \pi \rightarrow \mathbb{Z}$ be an epimorphism and $\mathbb{k}$ be a commutative ring. Identify the group-ring $\mathbb{k} A$ with the Laurent polynomial ring $\Lambda=\mathbb{k}\left[t^{ \pm 1}\right]$, and note that the augmentation ideal of $\Lambda$ is principal: $J=(t-1) \Lambda$. Also identify the associated graded ring $\operatorname{gr}(\mathbb{k} \mathbb{Z})$ with the polynomial ring $\mathbb{k}[x]$, where $x=t-1$. Assuming either $\mathbb{k}=\mathbb{Z}$ and $H_{*}(X, \mathbb{Z})$ is torsion-free, or $\mathbb{k}$ is a field, it follows from (20) and Lemma 6.1 that

$$
E_{-s, t}^{1}\left(X, \Lambda_{\nu}\right)=\operatorname{gr}_{J}^{s}(\mathbb{k} \mathbb{Z}) \otimes_{\mathbb{k}} H_{t-s}(X, \mathbb{k})=\mathbb{k} \otimes_{\mathbb{k}} H_{t-s}(X, \mathbb{k})=H_{t-s}(X, \mathbb{k}),
$$

where we used the fact that $\operatorname{gr}_{J}^{s}(\mathbb{k} \mathbb{Z})$ is freely generated by $x^{s}$.

The induced homomorphism $\nu_{*}: H_{1}(\pi, \mathbb{k}) \rightarrow H_{1}(\mathbb{Z}, \mathbb{k})=\mathbb{k}$ determines a cohomology class $\nu_{\mathbb{k}} \in H^{1}(X, \mathbb{k})$. Proceeding as in the proof of Proposition 7.3 , we obtain the following.

Corollary 8.3. Let $X$ be a connected $C W$-complex and let $\nu: \pi_{1}(X) \rightarrow \mathbb{Z}$ be an epimorphism. Assume either that $\mathbb{k}=\mathbb{Z}$ and $H_{*}(X, \mathbb{Z})$ is torsion-free, or $\mathbb{k}$ is a field. With identifications as in (38), the differential $d^{1}: E_{-s, s+q}^{1} \rightarrow E_{-s-1, s+q}^{1}$ is the composite

$$
H_{q}(X, \mathbb{k}) \stackrel{\nabla_{X}}{\longrightarrow} H_{1}(X, \mathbb{k}) \otimes_{\mathbb{k}} H_{q-1}(X, \mathbb{k}) \stackrel{\nu_{*} \otimes \mathrm{id}}{\longrightarrow} \mathbb{k} \otimes_{\mathbb{k}} H_{q-1}(X, \mathbb{k})=H_{q-1}(X, \mathbb{k}) .
$$

If, moreover, $X$ is of finite type, then the dual $\delta^{1}=\left(d^{1}\right)^{*}$ coincides with the leftmultiplication map

$$
\cdot \nu_{\mathbb{k}}: H^{q-1}(X, \mathbb{k}) \rightarrow H^{q}(X, \mathbb{k}) .
$$

8.3. Comparing the two filtrations. Next, identify the $J$-adic completion of $\Lambda=\mathbb{k} \mathbb{Z}$ with the power-series ring $\widehat{\Lambda}=\mathbb{k}[[x]]$ so that the map $\iota: \Lambda \rightarrow \widehat{\Lambda}$ sends $t$ to $x+1$. Note that the extension of the ideal $J$ is principal: $\widehat{J}=x \widehat{\Lambda}$.

Let $\nu: \pi \rightarrow \mathbb{Z}$ be an epimorphism. We have seen in 6.2 that each piece of the corresponding spectral sequences, ${ }_{q} E_{*}^{r}$ and ${ }_{q} \widehat{E}_{*}^{r}$, supports a natural graded module structure over the ring $\operatorname{gr}_{J}(\Lambda)=\operatorname{gr}_{\widehat{J}}(\widehat{\Lambda})$. Recall from Corollary 6.7 that, for any commutative ring $R$, viewed as a right $\mathbb{k} \pi$-module via a ring map $\rho: \mathbb{k} \pi \rightarrow R$, we have an inclusion $J^{s} \cdot H_{q}(X, R) \subseteq F_{-s} H_{q}(X, R)$, where $J$ is the ideal of $R$ generated by $\rho(I)$.

Lemma 8.4. Let $X$ be a connected $C W$-complex and $\mathbb{k}$ a commutative ring. Set $R=\mathbb{k} \mathbb{Z}_{\nu}$ or $\widehat{\mathbb{k}}_{\hat{\nu}}$. Then

(1) For each $r$ and $q$, the $\operatorname{gr}(R)$-module ${ }_{q} E_{*}^{r}$ is generated by ${ }_{q} E_{0}^{r}$.

(2) The spectral sequence filtration on $H_{*}(X, R)$ coincides with the $(x)$-adic filtration.

Proof. Part (11). It is enough to check that $Z_{-s}^{r}=x^{s} \cdot Z_{0}^{r}$ for all $r, s \geq 0$. Recall from (29) that $F^{k} C_{\bullet}(X, R)=x^{k} \cdot C_{\bullet}(X, R)$. If $z=x^{s} y$ and $\tilde{\partial}(y)=x^{r} y^{\prime}$, then $z \in F^{s}$ and $\tilde{\partial}(z)=x^{s+r} y^{\prime} \in F^{s+r}$, hence $z \in Z_{-s}^{r}$. Conversely, if $z=x^{s} y$ and $\tilde{\partial}(z)=x^{s} \tilde{\partial}(y)=x^{r+s} y^{\prime}$, then $\tilde{\partial}(y)=x^{r} y^{\prime}$, and therefore $y \in Z_{0}^{r}$, as needed.

Part (2) follows from the equality $Z_{-s}^{\infty}=x^{s} \cdot Z_{0}^{\infty}$, which is checked in a similar way.

Nevertheless, the filtration $F_{\bullet}(X, \mathbb{k} \mathbb{Z})$ need not be separated, as the next example shows. 
Example 8.5. Let $X=S^{1} \times\left(S^{1} \vee S^{1}\right)$, and identify $\pi=\pi_{1}(X)$ with $\mathbb{Z} \times F_{2}=$ $\langle a, b, c| a$ central $\rangle$. The epimorphism $\nu: \pi \rightarrow \mathbb{Z}$ that sends $a \mapsto 2, b \mapsto 1, c \mapsto 1$ defines a Galois $\mathbb{Z}$-cover, $Y \rightarrow X$. Identify the group ring $\mathbb{k} \mathbb{Z}$ with $\Lambda=\mathbb{k}\left[t^{ \pm 1}\right]$, and consider the $\Lambda$-module $N:=H_{1}(Y, \mathbb{k})=H_{1}\left(X, \mathbb{k} \mathbb{Z}_{\nu}\right)$. Then

$$
N= \begin{cases}(\Lambda /(1-t))^{2} \oplus \Lambda /(1+t) & \text { if char } \mathbb{k} \neq 2, \\ \Lambda /(1-t) \oplus \Lambda /(1-t)^{2} & \text { if char } \mathbb{k}=2 .\end{cases}
$$

Thus,

$$
\bigcap_{s \geq 0} J^{s} N= \begin{cases}\Lambda /(1+t) & \text { if } \operatorname{char} \mathbb{k} \neq 2, \\ 0 & \text { if } \operatorname{char} \mathbb{k}=2,\end{cases}
$$

where $J$ denotes the augmentation ideal of $\Lambda$. By Lemma 8.4, the filtration $\left\{F_{s} N\right\}$ is not separated, if char $\mathbb{k} \neq 2$. In this situation, the spectral sequence $E^{\bullet}(X, \mathbb{k} \mathbb{Z})$ converges, yet we cannot recover $N=H_{1}(X, \mathbb{k} \mathbb{Z})$ from the spectral sequence, even additively.

\section{Monodromy action and the Aomoto complex}

In the previous section we showed that the equivariant spectral sequence of a finite type $\mathrm{CW}$-complex $X$, starting at $E_{s, t}^{1}\left(X, \mathbb{k} \mathbb{Z}_{\nu}\right)$, converges to $H_{s+t}\left(X, \mathbb{k} \mathbb{Z}_{\nu}\right)$, the homology groups of the cover determined by an epimorphism $\nu: \pi_{1}(X) \rightarrow \mathbb{Z}$, with coefficients in a field $\mathbb{k}$. Using this fact, we now investigate the monodromy action of $\mathbb{Z}$ on $H_{*}\left(X, \mathbb{k} \mathbb{Z}_{\nu}\right)$. In the process, we relate the triviality of the action to the exactness of the Aomoto complex defined by $\nu_{\mathbb{k}} \in H^{1}(X, \mathbb{k})$.

9.1. Modules over $\mathbb{k} \mathbb{Z}$. Start by identifying the group-ring $\mathbb{k} \mathbb{Z}$ with $\Lambda=\mathbb{k}\left[t^{ \pm 1}\right]$. Since $\mathbb{k}$ is a field, the ring $\Lambda$ is a PID, and so every finitely-generated $\Lambda$-module $P$ decomposes as a direct sum of a free module, $P_{0}=\Lambda^{\operatorname{rank} P}$, with finitely many modules of the form

$$
P_{f}=\bigoplus_{i>0}\left(\Lambda / f^{i}\right)^{e_{f, i}(P)}
$$

indexed by irreducible polynomials $f \in \Lambda$. We call $P_{f}$ the $f$-primary part of $P$ and $\Lambda / f^{i}$ an $f$-primary Jordan block of size $i$.

Now let $X$ be a finite-type CW complex and $\nu: \pi_{1}(X) \rightarrow \mathbb{Z}$ be an epimorphism. For each $q \geq 0$, the homology group $P^{q}:=H_{q}\left(X, \mathbb{k} \mathbb{Z}_{\nu}\right)$, viewed as a module over $\Lambda=\mathbb{k} \mathbb{Z}$, decomposes as

$$
H_{q}\left(X, \mathbb{k} \mathbb{Z}_{\nu}\right)=P_{0}^{q} \oplus P_{t-1}^{q} \oplus \bigoplus_{f(1) \neq 0} P_{f}^{q},
$$

with $P_{0}^{q}=\Lambda^{r_{q}}$, where $r_{q}$ is the rank of $P^{q}$, and $P_{t-1}^{q}=\bigoplus_{i>0}\left(\Lambda /(t-1)^{i}\right)^{e_{i}^{q}}$, where $e_{i}^{q}=e_{t-1, i}\left(P^{q}\right)$ is the number of $(t-1)$-primary Jordan blocks of size $i$.

Our first result in this section identifies precisely the information that the $I$ adic spectral sequence carries about the $\mathbb{k} \mathbb{Z}$-module structure on $H_{*}\left(X, \mathbb{k} \mathbb{Z}_{\nu}\right)$, or, equivalently, the monodromy action of the group $\mathbb{Z}=\langle t\rangle$ on the homology groups of the covering space of $X$ determined by $\nu$. Set $J=(t-1) \mathbb{k} \mathbb{Z}$.

Proposition 9.1. For each $q \geq 0$, the $\operatorname{gr}_{J}(\mathbb{R} \mathbb{Z})$-module structure on ${ }_{q} E^{\infty}\left(X, \mathbb{k} \mathbb{Z}_{\nu}\right)$ described in 6.2 determines the free and $(t-1)$-primary parts of $H_{q}\left(X, \mathbb{k} \mathbb{Z}_{\nu}\right)$, viewed as a module over $\mathbb{k} \mathbb{Z}$. 
Proof. Identify $\operatorname{gr}_{J}(\mathbb{k} \mathbb{Z})=\mathbb{k}[x]$, where $x=t-1$. Proposition 8.1, together with Lemma 8.4 and (35), guarantee that the $\mathbb{k}[x]$-modules ${ }_{q} E^{\infty}$ and $\operatorname{gr}_{J} H_{q}\left(X, \mathbb{k} \mathbb{Z}_{\nu}\right)$ are isomorphic. From (40), we obtain a decomposition

$$
\operatorname{gr}_{J} H_{q}\left(X, \mathbb{k} \mathbb{Z}_{\nu}\right)=\mathbb{k}[x]^{r_{q}} \oplus \bigoplus_{i>0}\left(\mathbb{k}[x] / x^{i}\right)^{e_{i}^{q}}
$$

as $\mathbb{k}[x]$-modules. Hence, the $\operatorname{gr}_{J}(\mathbb{k} \mathbb{Z})$-module structure of ${ }_{q} E^{\infty}$ determines the numbers $r_{q}$ and $e_{i}^{q}$, that is, the free and $(t-1)$-primary parts of $H_{q}\left(X, \mathbb{k} \mathbb{Z}_{\nu}\right)$.

9.2. Aomoto complex. As before, denote by $\nu_{\mathbb{Z}}$ the class in $H^{1}(X, \mathbb{Z})$ determined by an arbitrary homomorphism $\nu: \pi \rightarrow \mathbb{Z}$. Let $\nu_{*}: H_{1}(X, \mathbb{k}) \rightarrow H_{1}(\mathbb{Z}, \mathbb{k})=\mathbb{k}$ be the induced homomorphism. The corresponding cohomology class, $\nu_{\mathbb{k}} \in H^{1}(X, \mathbb{k})$, is the image of $\nu_{\mathbb{Z}}$ under the coefficient homomorphism $\mathbb{Z} \rightarrow \mathbb{k}$. We then have

$$
\nu_{\mathbb{k}} \cup \nu_{\mathbb{k}}=0 \text { in } H^{2}(X, \mathbb{k}) .
$$

Indeed, by obstruction theory, there is a map $f: X \rightarrow S^{1}$ and a class $\omega \in H^{1}\left(S^{1}, \mathbb{Z}\right)$ such that $\nu_{\mathbb{Z}}=f^{*}(\omega)$. Hence, $\nu_{\mathbb{Z}} \cup \nu_{\mathbb{Z}}=f^{*}(\omega \cup \omega)=0$. Formula (42) then follows by naturality of cup products with respect to coefficient homomorphisms.

As a consequence, left-multiplication by $\nu_{\mathbb{k}}$ turns the cohomology $\operatorname{ring} H^{*}(X, \mathbb{k})$ into a cochain complex,

$$
\left(H^{*}(X, \mathbb{k}), \nu_{\mathbb{k}}\right): H^{0}(X, \mathbb{k}) \stackrel{\nu_{\mathrm{k}}}{\longrightarrow} H^{1}(X, \mathbb{k}) \stackrel{\nu_{\mathrm{k}}}{\longrightarrow} H^{2}(X, \mathbb{k}) \longrightarrow \cdots,
$$

which we call the Aomoto complex of $H^{*}(X, \mathbb{k})$ with respect to $\nu_{\mathbb{k}}$.

Definition 9.2. The Aomoto Betti numbers of $X$, with respect to the cohomology class $\nu_{\mathbb{k}} \in H^{1}(X, \mathbb{k})$, are defined as

$$
\beta_{q}\left(X, \nu_{\mathbb{k}}\right):=\operatorname{dim}_{\mathbb{k}} H^{q}\left(H^{*}(X, \mathbb{k}), \cdot \nu_{\mathbb{k}}\right) .
$$

Clearly, $\beta_{q}\left(X, \nu_{\mathbb{k}}\right) \leq \operatorname{dim}_{\mathbb{k}} H^{q}(X, \mathbb{k})$. In general, though, the inequality is strict.

Example 9.3. Let $X=T^{n}$, the $n$-dimensional torus. The cohomology ring $H^{*}(X, \mathbb{k})$ is simply the exterior algebra on $H^{1}(X, \mathbb{k})=\mathbb{k}^{n}$. If $\nu$ is onto, then $\nu_{\mathbb{k}} \neq 0$, and so the complex (43) is exact. Thus, $\beta_{q}\left(X, \nu_{\mathbb{k}}\right)=0$ for all $q \geq 0$, though, of course, $b_{q}(X)=\left(\begin{array}{l}n \\ q\end{array}\right)$.

9.3. Monodromy action. We are now ready to state and prove the second result of this section. Let $P^{q}=H_{q}\left(X, \mathbb{k} \mathbb{Z}_{\nu}\right)$, viewed as a module over $\mathbb{k} \mathbb{Z}$ via the epimorphism $\nu: \pi \rightarrow \mathbb{Z}$, with decomposition as in (40).

Proposition 9.4. For each $k \geq 0$, the following are equivalent:

(1) For each $q \leq k$, the vector space $H_{q}\left(X, \mathbb{k} \mathbb{Z}_{\nu}\right)$ is finite-dimensional and contains no $(t-1)$-primary Jordan blocks of size greater than 1 .

(2) For each $q \leq k$, the monodromy action of $\mathbb{k} \mathbb{Z}$ on $P_{0}^{q} \oplus P_{t-1}^{q}$ is trivial.

(3) $\beta_{0}\left(X, \nu_{\mathbb{k}}\right)=\cdots=\beta_{k}\left(X, \nu_{\mathbb{k}}\right)=0$.

Proof. The equivalence (11) $\Leftrightarrow$ (2) is an immediate consequence of decompositions (39)-(40). To prove the equivalence (2) $\Leftrightarrow(3)$, recall that we have a spectral sequence $E_{-p, q}^{1}=\operatorname{gr}^{p}(\mathbb{k} \mathbb{Z}) \otimes H_{q-p}(X, \mathbb{k}) \Rightarrow H_{q}\left(X, \mathbb{k}_{\nu}\right)$ whose differential $d^{1}: H_{q}(X, \mathbb{k}) \rightarrow H_{q-1}(X, \mathbb{k})$ is the transpose of $\cdot \nu_{\mathbb{k}}$. 
(3) $\Rightarrow$ (2). The condition $\beta_{\leq k}\left(X, \nu_{\mathbb{k}}\right)=0$ is equivalent to $E_{-p, q}^{2}=0$, for $q-p \leq k$ and $p>0$. Consequently, $E_{-p, q}^{\infty}=0$, in the same range. By convergence of the spectral sequence and Lemma 8.4 , this means that

$$
\frac{(t-1)^{p} \cdot H_{q-p}\left(X, \mathbb{k} \mathbb{Z}_{\nu}\right)}{(t-1)^{p+1} \cdot H_{q-p}\left(X, \mathbb{k} \mathbb{Z}_{\nu}\right)}=0, \quad \text { for } q-p \leq k \text { and } p>0 .
$$

Taking $p=1$ in the above, and recalling the discussion from 9.1, we obtain

$$
\frac{(t-1) \cdot\left(P_{0}^{q} \oplus P_{t-1}^{q}\right)}{(t-1)^{2} \cdot\left(P_{0}^{q} \oplus P_{t-1}^{q}\right)}=0
$$

for all $q \leq k$. This implies $r_{q}=0\left(\right.$ since $\operatorname{gr}^{1}(\Lambda) \neq 0$ ) and $e_{i}^{q}=0$, for $i>1$ (since $\left.\operatorname{dim}_{\mathbb{k}} \operatorname{gr}^{1}\left(P_{t-1}^{q}\right)=\sum_{i>1} e_{i}^{q}\right)$ and for all $q \leq k$, which is equivalent to $t-1=0$ on $P_{0}^{q} \oplus P_{t-1}^{q}$.

(2) $\Rightarrow$ (3). Induction on $k$. For $k=0$, we have

$$
\beta_{0}\left(X, \nu_{\mathbb{k}}\right)=\operatorname{dim}_{\mathbb{k}} \operatorname{ker}\left(\cdot \nu_{\mathbb{k}}: H^{0}(X, \mathbb{k}) \rightarrow H^{1}(X, \mathbb{k})\right)=0 .
$$

Now assume $\beta_{\leq k-1}\left(X, \nu_{\mathbb{k}}\right)=0$. Then $E_{-1, k+1}^{2}=\cdots=E_{-1, k+1}^{\infty}$. Moreover, we always have $\beta_{q}\left(X, \nu_{\mathbb{k}}\right)=\operatorname{dim}_{\mathbb{k}} E_{-p, q+p}^{2}$, for all $p \geq 1$. In our situation, we have

$$
\beta_{k}\left(X, \nu_{\mathbb{k}}\right)=\operatorname{dim}_{\mathbb{k}} E_{-1, k+1}^{\infty}=\operatorname{dim}_{\mathbb{k}} \frac{(t-1) \cdot\left(P_{0}^{k} \oplus P_{t-1}^{k}\right)}{(t-1)^{2} \cdot\left(P_{0}^{k} \oplus P_{t-1}^{k}\right)},
$$

and this vanishes, by assumption. The induction step is thus proved.

\section{BOUNDS ON TWISTED COHOMOLOGY RANKS: I}

In this section, we give upper bounds on the ranks of the cohomology groups with coefficients in a rank 1 local system defined by a rational character of prime-power order.

10.1. Twisted Betti numbers. We start with some definitions. Let $X$ be a connected, finite-type CW-complex. Given a field $\mathbb{k}$, the $\mathbb{k}$-Betti numbers of $X$ are defined as $b_{q}(X, \mathbb{k}):=\operatorname{dim}_{\mathbb{k}} H^{q}(X, \mathbb{k})$. More generally, if $R$ is a Noetherian ring, define $b_{q}(X, R)$ to be the minimal number of generators of the $R$-module $H^{q}(X, R)$.

Now suppose $\rho: \pi \rightarrow \mathbb{C}^{\times}$is a homomorphism from $\pi=\pi_{1}(X)$ to the multiplicative group of non-zero complex numbers. The twisted Betti numbers of $X$ corresponding to the character $\rho$ are defined by

$$
b_{q}(X, \rho):=\operatorname{dim}_{\mathbb{C}} H^{q}\left(X,{ }_{\rho} \mathbb{C}\right),
$$

where ${ }_{\rho} \mathbb{C}=\mathbb{C}$, viewed as a left module over $\mathbb{C} \pi$, via $\rho$. By duality, $b_{q}(X, \rho)=$ $\operatorname{dim}_{\mathbb{C}} H_{q}\left(X, \mathbb{C}_{\rho}\right)$, where $\mathbb{C}_{\rho}=\mathbb{C}$, viewed as a right module over $\mathbb{C} \pi$, via $\rho$.

Of particular importance to us are characters of the form $\rho(g)=\zeta^{\nu(g)}$, where $\nu: \pi \rightarrow \mathbb{Z}$ is a homomorphism, $d$ is a positive integer, and $\zeta$ is a primitive $d$-th root of unity. In this case, we say $\rho$ is a rational character of order $d$ and write

$$
b_{q}(X, \nu / d):=b_{q}(X, \rho) .
$$


It follows from elementary Galois theory that the twisted Betti numbers $b_{q}(X, \nu / d)$ do not depend on the choice of primitive $d$-th root of unity, thereby justifying the notation.

10.2. Cyclotomic polynomials. For each positive integer $d$, the $d$-th cyclotomic polynomial is defined as $\Phi_{d}(t)=\prod_{\zeta}(t-\zeta)$, where $\zeta$ ranges over all primitive $d$-th roots of unity. If $d=p^{r}$, with $p$ a prime, then $\Phi_{d}(t)=\left(t^{p^{r}}-1\right) /\left(t^{p^{r-1}}-1\right)$, and so $\Phi_{d}(1)=p$. If $d$ is not a prime power, and $d>1$, then $\Phi_{d}(1)=1$.

Lemma 10.1. Let $Q \in \mathbb{Z}[t]$ be a polynomial with integer coefficients. Suppose $Q(\zeta)=0$, for some root of unity $\zeta \in \mathbb{C}$ of prime-power order $p^{r}$. Then $Q(1)=0$ $(\bmod p)$.

Proof. The minimal polynomial of $\zeta$ is the cyclotomic polynomial $\Phi_{p^{r}}(t)$. Thus, $\Phi_{p^{r}} \mid Q$. But $\Phi_{p^{r}}(1)=p$, and so $p \mid Q(1)$.

The following corollary will be useful in the sequel. Let $A$ be a matrix with entries in $\mathbb{Z}\left[t^{ \pm 1}\right]$. We will denote by $A(z)$ the evaluation of $A$ at a non-zero complex number $z \in \mathbb{C}^{\times}$. For $p$ a prime, we may also view $A(1)$, after reduction modulo $p$, as a matrix with entries in $\mathbb{F}_{p}$.

Corollary 10.2. Let $\zeta$ be a root of unity of order a power of a prime $p$. Then

$$
\operatorname{rank}_{\mathbb{C}} A(\zeta) \geq \operatorname{rank}_{\mathbb{F}_{p}} A(1) .
$$

Proof. Suppose $m(t)$ is a minor of $A$, and $m(\zeta)=0$. Then $t^{k} m(t) \in \mathbb{Z}[t]$, for some $k \geq 0$, and $\zeta^{k} m(\zeta)=0$. Hence, by Lemma $10.1 m(1)=0$ in $\mathbb{F}_{p}$. The conclusion follows.

10.3. Modular Betti bounds. The following result relates the two kinds of Betti numbers defined above, under a prime-power assumption on the order of the rational character.

Theorem 10.3. Let $X$ be a connected, finite-type $C W$-complex. Let $\nu: \pi_{1}(X) \rightarrow \mathbb{Z}$ be a homomorphism and $p$ be a prime. Then, for all $r \geq 1$ and $q \geq 0$,

$$
b_{q}\left(X, \nu / p^{r}\right) \leq b_{q}\left(X, \mathbb{F}_{p}\right) .
$$

Proof. Let $C_{\bullet}(X, \mathbb{Z})=\left(C_{q}, \partial_{q}\right)_{q \geq 0}$ be the cellular chain complex of $X$, and let $\mathbb{Z} \mathbb{Z}_{\nu}$ be the ring $\mathbb{Z Z}=\mathbb{Z}\left[t^{ \pm 1}\right]$, viewed as a module over $\mathbb{Z} \pi_{1}(X)$ via the ring map $\bar{\nu}: \mathbb{Z} \pi_{1}(X) \rightarrow \mathbb{Z} \mathbb{Z}$. The equivariant chain complex $C_{\bullet}\left(X, \mathbb{Z} \mathbb{Z}_{\nu}\right)$ has chains $C_{q}\left(X, \mathbb{Z} \mathbb{Z}_{\nu}\right)=\mathbb{Z}\left[t^{ \pm 1}\right] \otimes C_{q}$ and differentials

$$
\partial_{q}^{\nu}:=\tilde{\partial}_{q}^{\mathbb{Z} \mathbb{Z}_{\nu}}: \mathbb{Z}\left[t^{ \pm 1}\right] \otimes C_{q} \rightarrow \mathbb{Z}\left[t^{ \pm 1}\right] \otimes C_{q-1} .
$$

Note that $\partial_{q}^{\nu}(1)=\partial_{q}$. From the definition of twisted Betti numbers, we have

$$
b_{q}\left(X, \nu / p^{r}\right)=\operatorname{rank} C_{q}-\operatorname{rank}_{\mathbb{C}} \partial_{q}^{\nu}(\zeta)-\operatorname{rank}_{\mathbb{C}} \partial_{q+1}^{\nu}(\zeta),
$$

where $\zeta$ is a primitive root of unity of order $p^{r}$.

Now consider the chain complex $C \cdot\left(X, \mathbb{F}_{p}\right)$. By definition, $C_{q}\left(X, \mathbb{F}_{p}\right)=C_{q} \otimes \mathbb{F}_{p}$. Clearly, the differential $\partial_{q} \otimes \operatorname{id}_{\mathbb{F}_{p}}$ equals the reduction mod $p$ of $\partial_{q}^{\nu}(1)$. Thus,

$$
b_{q}\left(X, \mathbb{F}_{p}\right)=\operatorname{rank} C_{q}-\operatorname{rank}_{\mathbb{F}_{p}} \partial_{q}^{\nu}(1)-\operatorname{rank}_{\mathbb{F}_{p}} \partial_{q+1}^{\nu}(1)
$$

The desired inequality follows from (49), (50), and Corollary 10.2 
Remark 10.4. Given a knot $K$ in $S^{3}$, let $X=S^{3} \backslash K$ be the knot complement, $\pi=\pi_{1}(X)$ the knot group, and $\nu=\mathrm{ab}: \pi \rightarrow \mathbb{Z}$ the abelianization map. The Alexander polynomial of the knot, $\Delta_{K}(t) \in \mathbb{Z}\left[t^{ \pm 1}\right]$, is the greatest common divisor of the codimension 1 minors of the Alexander matrix, $\partial_{2}^{\nu}$, defined as in (48). Let $\rho: \pi \rightarrow \mathbb{C}^{\times}$be a non-trivial character. Writing $\rho(g)=z^{\nu(g)}$, for some $z \in \mathbb{C}^{\times}$, we have

$$
b_{1}(X, \rho) \neq 0 \Longleftrightarrow \Delta_{K}(z)=0
$$

(see 9] for a much more general statement). In particular, $b_{1}(X, \nu / d) \neq 0$ if and only if $\Delta_{K}(\zeta)=0$, for some primitive $d$-th root of unity $\zeta$.

10.4. Examples and discussion. We conclude this section by discussing the necessity of the hypothesis in Theorem 10.3, and the sharpness of inequality (47).

We start with the prime-power hypothesis. Suppose $d$ is a positive integer so that $d \neq p^{r}$, for any prime $p$. One may wonder whether an inequality of the form

$$
b_{q}(X, \nu / d) \leq b_{q}(X, R)
$$

holds, for some suitable choice of Noetherian ring $R$. The following example shows that this is not possible, in general.

Example 10.5. By assumption, $d$ is not a prime-power integer; thus, $\Phi_{d}(1)=1$. On the other hand, $\Phi_{d}\left(t^{-1}\right) \equiv \Phi_{d}(t)$, up to units in $\mathbb{Z}\left[t^{ \pm 1}\right]$. Hence, by a classical result of Seifert [29], there is a knot $K_{d}$ in $S^{3}$ with Alexander polynomial $\Delta_{K_{d}}(t)=$ $\Phi_{d}(t)$.

Fix an integer $n>1$, and let $K=\sharp^{n} K_{d}$ be the connected sum of $n$ copies of $K_{d}$. Denote by $X_{d}=S^{3} \backslash K_{d}$ and $X=S^{3} \backslash K$ the corresponding knot complements. By additivity of Alexander invariants under connected sums of knots (see for instance [28), we have an isomorphism of $\mathbb{C} Z$-modules, $H_{1}\left(X, \mathbb{C Z}_{\nu}\right) \cong H_{1}\left(X_{d}, \mathbb{C Z}_{\nu_{d}}\right)^{n}$, where the twisted coefficients are defined by abelianization. Let $\mathbb{C}_{\zeta}=\mathbb{C}$, viewed as a $\mathbb{C Z}$-module via evaluation of Laurent polynomials at a primitive $d$-root of unity $\zeta$. Then

$$
\begin{aligned}
b_{1}(X, \nu / d) & =\operatorname{dim}_{\mathbb{C}}\left(\mathbb{C}_{\zeta} \otimes_{\mathbb{C Z}} H_{1}\left(X, \mathbb{C Z}_{\nu}\right)\right) \\
& =n \operatorname{dim}_{\mathbb{C}}\left(\mathbb{C}_{\zeta} \otimes_{\mathbb{C Z}} H_{1}\left(X_{d}, \mathbb{C Z}_{\nu_{d}}\right)\right) \\
& =n b_{1}\left(X_{d}, \nu_{d} / d\right) .
\end{aligned}
$$

But $\Delta_{K_{d}}(\zeta)=\Phi_{d}(\zeta)=0$, and so, as noted in Remark 10.4, $b_{1}\left(X_{d}, \nu_{d} / d\right) \geq 1$. Thus, $b_{1}(X, \nu / d) \geq n$. On the other hand, $b_{1}(X, R)=1$, for any ring $R$.

Next, we show that inequality (47) from Theorem 10.3 may fail to be an equality.

Example 10.6. Let $X=S^{3} \backslash K$ be a knot complement, $\nu: \pi_{1}(X) \rightarrow \mathbb{Z}$ the abelianization map, and $d=p^{r}$. If $b_{1}(X, \nu / d)$ were non-zero, then $\Delta_{K}(\zeta)$ would vanish, and so $\Phi_{d}(t)$ would divide $\Delta_{K}(t)$. But $\Phi_{d}(1)=p$, while $\Delta_{K}(1)= \pm 1$. Hence, we have $b_{1}(X, \nu / d)=0$, yet $b_{1}\left(X, \mathbb{F}_{p}\right)=1$.

Finally, we show that the bound (47) cannot be improved.

Example 10.7. Let $X$ be any connected, finite-type CW-complex with $H_{q}(X, \mathbb{Z})$ torsion-free, and let $\nu: \pi_{1}(X) \rightarrow \mathbb{Z}$ be the zero map. Then $b_{q}\left(X, \nu / p^{r}\right)=b_{q}\left(X, \mathbb{F}_{p}\right)$ $=b_{q}(X)$, for all primes $p$. 


\section{BOUNDS ON TWISTED COHOMOLOGY RANKS: II}

Continuing the theme from the previous section, we sharpen the bounds on the twisted Betti numbers $b_{q}\left(X, \nu / p^{r}\right)$ by using the Aomoto Betti numbers $\beta_{q}\left(X, \nu_{\mathbb{F}_{p}}\right)$ instead of the usual Betti numbers $b_{q}\left(X, \mathbb{F}_{p}\right)$, under an additional freeness assumption on $H_{*}(X, \mathbb{Z})$.

11.1. Decomposing the boundary map. Let $X$ be a connected, finite-type CWcomplex. Assume $\mathbb{k}=\mathbb{Z}$ and $H_{*}(X, \mathbb{Z})$ is torsion-free, or $\mathbb{k}$ is a field. Let $C_{q}=$ $C_{q}(X, \mathbb{k})$ be the the group of cellular $q$-chains over $\mathbb{k}$ and $\partial_{q}: C_{q} \rightarrow C_{q-1}$ be the boundary map. Writing $Z_{q}=\operatorname{ker} \partial_{q}$ and $B_{q-1}=\operatorname{im} \partial_{q}$, we have a split exact sequence

$$
0 \rightarrow Z_{q} \rightarrow C_{q} \stackrel{\partial_{q}}{\rightarrow} B_{q-1} \rightarrow 0 .
$$

By assumption, $H_{q}=Z_{q} / B_{q}$ is a free $\mathbb{k}$-module. Thus,

$$
C_{q} \cong Z_{q} \oplus B_{q-1} \cong B_{q} \oplus N_{q},
$$

where $N_{q} \cong H_{q} \oplus B_{q-1}$. The next lemma follows at once.

Lemma 11.1. With respect to the direct sum decompositions $C_{q}=Z_{q} \oplus B_{q-1}$ and $C_{q-1}=B_{q-1} \oplus N_{q-1}$, the matrix of the differential $\partial_{q}: C_{q} \rightarrow C_{q-1}$ takes the block-matrix form

$$
\partial_{q}=\left(\begin{array}{cc}
0 & \mathrm{id} \\
0 & 0
\end{array}\right)
$$

Remark 11.2. Suppose $H_{*}(X, \mathbb{Z})$ is torsion-free. Then, by standard homological algebra, $Z_{q}(X, \mathbb{k})=Z_{q}(X, \mathbb{Z}) \otimes \mathbb{k}, B_{q}(X, \mathbb{k})=B_{q}(X, \mathbb{Z}) \otimes \mathbb{k}$, and $H_{q}(X, \mathbb{k})=$ $H_{q}(X, \mathbb{Z}) \otimes \mathbb{k}$. It easily follows that the block-matrix decomposition (54) of $\partial_{q}$ is compatible with the canonical coefficient homomorphism $\mathbb{Z} \rightarrow \mathbb{k}$.

11.2. Aomoto bounds on the twisted Betti numbers. We are now ready to state the main result of this section.

Theorem 11.3. Let $X$ be a connected, finite-type $C W$-complex, with $H_{*}(X, \mathbb{Z})$ torsion-free. Let $\nu: \pi_{1}(X) \rightarrow \mathbb{Z}$ be a homomorphism and $p$ be a prime. Then, for all $r \geq 1$ and $q \geq 0$,

$$
b_{q}\left(X, \nu / p^{r}\right) \leq \beta_{q}\left(X, \nu_{\mathbb{F}_{p}}\right) .
$$

Proof. Without loss of generality, we may assume $\nu$ is surjective. Indeed, if $\nu=0$, then clearly

$$
b_{q}\left(X, \nu / p^{r}\right)=b_{q}(X, \mathbb{C}) \leq b_{q}\left(X, \mathbb{F}_{p}\right)=\beta_{q}\left(X, \nu_{\mathbb{F}_{p}}\right) .
$$

On the other hand, if the image of $\nu$ has index $m$ in $\mathbb{Z}$, then plainly $b_{q}\left(X, \nu / p^{r}\right)=$ $b_{q}\left(X, \nu^{\prime} / p^{s}\right)$ for an epimorphism $\nu^{\prime}: \pi_{1}(X) \rightarrow \mathbb{Z}$ such that $\nu=m \nu^{\prime}$. (Here $p^{s}$ is the order of $\zeta^{m}$, where $\zeta$ is a primitive root of unity of order $p^{r}$.) If $p$ divides $m$, then $\nu_{\mathbb{F}_{p}}=0$ and claim (55) becomes inequality (47). Otherwise, $s \geq 1$ and $\beta_{q}\left(X, \nu_{\mathbb{F}_{p}}\right)=\beta_{q}\left(X, \nu_{\mathbb{F}_{p}}^{\prime}\right)$. Hence, the result for $\nu^{\prime}$ implies the claim for $\nu$. 
Consider the equivariant chain complex $C \cdot\left(X, \mathbb{Z}_{\nu}\right)$, viewed as a chain complex over $\Lambda=\mathbb{Z}\left[t^{ \pm 1}\right]$. Identify $C_{q}\left(X, \mathbb{Z}_{\nu}\right)=\Lambda \otimes C_{q}$, where $C_{q}=C_{q}(X, \mathbb{Z})$, and denote by $\partial_{q}^{\nu}: \Lambda \otimes C_{q} \rightarrow \Lambda \otimes C_{q-1}$ the boundary maps.

Since $\partial_{q}^{\nu}(1)=\partial_{q}$ and $\left.\partial_{q}\right|_{Z_{q}}=0$, the restriction of $\partial_{q}^{\nu}$ to $\Lambda \otimes Z_{q}$ takes values in $J \otimes C_{q-1}$, where $J=(t-1) \Lambda$. As in 11.1 write $C_{q}=Z_{q} \oplus B_{q-1}$ and $C_{q-1}=$ $N_{q-1} \oplus B_{q-1}$. Using formula (54), we see that $\partial_{q}^{\nu}$ takes the block-matrix form

$$
\partial_{q}^{\nu}=\left(\begin{array}{cc}
(t-1) \mathrm{P} & \mathrm{Q} \\
(t-1) \mathrm{R} & \mathrm{S}
\end{array}\right)
$$

where $\mathrm{Q}(1)$ is the identity and $\mathrm{S}(1)$ is the zero matrix. For future use, define

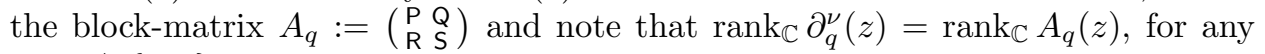
$z \in \mathbb{C} \backslash\{0,1\}$.

Recall from (49) that $b_{q}\left(X, \nu / p^{r}\right)=\operatorname{rank} C_{q}-\operatorname{rank}_{\mathbb{C}} \partial_{q}^{\nu}(\zeta)-\operatorname{rank}_{\mathbb{C}} \partial_{q+1}^{\nu}(\zeta)$, where $\zeta$ is a primitive root of unity of order $p^{r}$. Hence,

$$
b_{q}\left(X, \nu / p^{r}\right)=\operatorname{rank} C_{q}-\operatorname{rank}_{\mathbb{C}} A_{q}(\zeta)-\operatorname{rank}_{\mathbb{C}} A_{q+1}(\zeta) .
$$

Let us estimate the right side. By Corollary 10.2 .

$$
\operatorname{rank}_{\mathbb{C}} A_{q}(\zeta) \geq \operatorname{rank}_{\mathbb{F}_{p}} A_{q}(1)
$$

By Lemma 11.1 and Remark 11.2 ,

$$
\operatorname{rank}_{\mathbb{F}_{p}} A_{q}(1)=\operatorname{rank}_{\mathbb{F}_{p}} \mathrm{R}_{q}(1)+\operatorname{rank} B_{q-1} .
$$

Combining (57), (58), and (59) with the equality $b_{q}(X)=\operatorname{rank} C_{q}-\operatorname{rank} B_{q}-$ rank $B_{q-1}$, we obtain

$$
b_{q}\left(X, \nu / p^{r}\right) \leq b_{q}(X)-\operatorname{rank}_{\mathbb{F}_{p}} \mathrm{R}_{q}(1)-\operatorname{rank}_{\mathbb{F}_{p}} \mathrm{R}_{q+1}(1) .
$$

We now turn to the Aomoto-Betti numbers. By definition,

$$
\beta_{q}\left(X, \nu_{\mathbb{F}_{p}}\right)=b_{q}\left(X, \mathbb{F}_{p}\right)-\operatorname{rank}_{\mathbb{F}_{p}} \nu_{\mathbb{F}_{p}}^{q}-\operatorname{rank}_{\mathbb{F}_{p}} \nu_{\mathbb{F}_{p}}^{q+1},
$$

where $\nu_{\mathbb{F}_{p}}^{q}: H^{q-1}\left(X, \mathbb{F}_{p}\right) \rightarrow H^{q}\left(X, \mathbb{F}_{p}\right)$ denotes left-multiplication by $\nu_{\mathbb{F}_{p}} \in H^{1}\left(X, \mathbb{F}_{p}\right)$. By Corollary 8.3. this map is dual to the differential $d_{q}^{1}: E_{0, q}^{1} \rightarrow E_{-1, q}^{1}$, where $E_{0, q}^{1}=\operatorname{gr}^{0}\left(\mathbb{F}_{p} \mathbb{Z}\right) \otimes_{\mathbb{F}_{p}} H_{q}\left(X, \mathbb{F}_{p}\right)$ and $E_{-1, q}^{1}=\operatorname{gr}^{1}\left(\mathbb{F}_{p} \mathbb{Z}\right) \otimes_{\mathbb{F}_{p}} H_{q-1}\left(X, \mathbb{F}_{p}\right)$. Using the identifications $\operatorname{gr}^{0}\left(\mathbb{F}_{p} \mathbb{Z}\right)=\mathbb{F}_{p}$ and $\operatorname{gr}^{1}\left(\mathbb{F}_{p} \mathbb{Z}\right)=x \cdot \mathbb{F}_{p} \cong \mathbb{F}_{p}$, where $x=t-1$, we may view this differential as a map $d_{q}^{1}: H_{q}\left(X, \mathbb{F}_{p}\right) \rightarrow H_{q-1}\left(X, \mathbb{F}_{p}\right)$.

Clearly, $d_{q}^{1}$ has the same rank as $\delta_{q}^{1}=\iota \circ d_{q}^{1} \circ \pi$, where $\pi: Z_{q}\left(X, \mathbb{F}_{p}\right) \rightarrow H_{q}\left(X, \mathbb{F}_{p}\right)$ is the projection and $\iota: H_{q-1}\left(X, \mathbb{F}_{p}\right) \hookrightarrow N_{q-1}\left(X, \mathbb{F}_{p}\right)$ is the inclusion. Hence,

$$
\beta_{q}\left(X, \nu_{\mathbb{F}_{p}}\right)=b_{q}(X)-\operatorname{rank}_{\mathbb{F}_{p}} \delta_{q}^{1}-\operatorname{rank}_{\mathbb{F}_{p}} \delta_{q+1}^{1}
$$

In view of (600) and (62), it suffices to show that $\delta_{q}^{1}=\mathrm{R}_{q}(1)$. Let $z \in Z_{q}\left(X, \mathbb{F}_{p}\right)$. Using formula (56) - with everything reduced $\bmod p$-we find that

$$
\begin{aligned}
\delta_{q}^{1}(z) & =\iota\left(\left[\partial_{q}^{\nu}(1 \otimes z) \bmod J^{2}\right]\right) \\
& =(t-1) \mathrm{R}_{q}(t) z \bmod J^{2} \\
& =x \mathrm{R}_{q}(1) z \bmod J^{2} \\
& \equiv \mathrm{R}_{q}(1) z,
\end{aligned}
$$

where at the last step we used the identification $\operatorname{gr}^{1}\left(\mathbb{F}_{p} \mathbb{Z}\right)=J / J^{2}=x \cdot \mathbb{F}_{p} \cong \mathbb{F}_{p}$. This finishes the proof. 
11.3. Necessity of the hypothesis. We now give examples showing that the two hypotheses in Theorem 11.3 are necessary. We start with the prime-power hypothesis.

Given a Noetherian ring $R$, let $\nu_{R} \in H^{1}(X, R)$ be the cohomology class determined by the homomorphism $\pi_{1}(X) \stackrel{\nu}{\rightarrow} \mathbb{Z} \stackrel{\iota}{\rightarrow} R$, where $\iota(1)=1$. Define $\beta_{q}\left(X, \nu_{R}\right)$ to be the minimal number of generators of the $R$-module $H^{q}\left(H^{*}(X, R), \cdot \nu_{R}\right)$.

Now suppose $d$ is a positive integer so that $d \neq p^{r}$, for any prime $p$. One may wonder whether an inequality of the form $b_{q}(X, \nu / d) \leq \beta_{q}\left(X, \nu_{R}\right)$ holds, for some suitable choice of Noetherian ring $R$. The following example shows that this is not possible.

Example 11.4. Let us start by recalling an old result of R. Lyndon (see 1, Thm. 3.6]). If $v_{1}, \ldots, v_{n}$ are elements in $\Lambda=\mathbb{Z}^{n}$ satisfying $\sum_{i=1}^{n} v_{i}\left(t_{i}-1\right)=0$, then there is a word $r \in F_{n}^{\prime}$ such that $\left(\partial r / \partial x_{i}\right)^{\text {ab }}=v_{i}$, for all $i$. Hence, if $\pi=\left\langle x_{1}, \ldots, x_{n} \mid r\right\rangle$ is the corresponding 1-relator group and $X$ is the presentation 2-complex, then the chain complex $C_{\bullet}\left(X^{\mathrm{ab}}, \mathbb{k}\right)$ has boundary maps $\widetilde{\partial}_{2}^{\mathrm{ab}}: \Lambda \rightarrow \Lambda^{n}$ and $\widetilde{\partial}_{1}^{\text {ab }}: \Lambda^{n} \rightarrow \Lambda$ given by

$$
\widetilde{\partial}_{2}^{\mathrm{ab}}\left(1 \otimes e_{2}\right)=\sum_{i=1}^{n} v_{i} e_{1}^{i} \quad \text { and } \quad \widetilde{\partial}_{1}^{\mathrm{ab}}\left(e_{1}^{i}\right)=\left(t_{i}-1\right) e_{0} .
$$

Now take $v_{1}=\Phi_{d}\left(t_{1}\right)\left(t_{2}-1\right)$ and $v_{2}=\Phi_{d}\left(t_{1}\right)\left(1-t_{1}\right)$, and let $X$ be the 2 -complex constructed above. Let $\nu: \pi \rightarrow \mathbb{Z}, \nu\left(x_{1}\right)=\nu\left(x_{2}\right)=1$, and fix a primitive $d$-th root of unity $\zeta$. The chain complex $C \cdot\left(X, \mathbb{C}_{\rho}\right): \mathbb{C} \rightarrow \mathbb{C}^{2} \rightarrow \mathbb{C}$ corresponding to the character $\rho: \pi \rightarrow \mathbb{C}^{\times}, \rho(g)=\zeta^{\nu(g)}$, has boundary maps $\partial_{2}^{\rho}=0$ and $\partial_{1}^{\rho}=\left(\begin{array}{l}\zeta-1 \\ \zeta-1\end{array}\right)$. Hence, $H_{1}\left(X, \mathbb{C}_{\rho}\right)=\mathbb{C}$, and so $b_{1}(X, \nu / d)=1$.

Next, let $R$ be a Noetherian ring and set $J=I_{R} \mathbb{Z}^{2}$. The differential $d^{1}: H_{2}(X, R)$ $\rightarrow H_{1}(X, R) \otimes H_{1}(X, R)$ on $E^{1}\left(X, R \mathbb{Z}_{\mathrm{ab}}^{2}\right)$ is given by:

$$
\begin{aligned}
d^{1}\left(\llbracket e_{2} \rrbracket\right) & =\widetilde{\partial}_{2}^{\mathrm{ab}}\left(1 \otimes e_{2}\right) \quad \bmod J^{2} \\
& =\Phi_{d}\left(t_{1}\right)\left(t_{2}-1\right) \otimes \llbracket e_{1}^{1} \rrbracket+\Phi_{d}\left(t_{1}\right)\left(1-t_{1}\right) \otimes \llbracket e_{1}^{2} \rrbracket \quad \bmod J^{2} \\
& =\Phi_{d}(1)\left(t_{2}-1\right) \otimes \llbracket e_{1}^{1} \rrbracket+\Phi_{d}(1)\left(1-t_{1}\right) \otimes \llbracket e_{1}^{2} \rrbracket \quad \bmod J^{2} \\
& \equiv \Phi_{d}(1)\left(\llbracket e_{1}^{2} \rrbracket \otimes \llbracket e_{1}^{1} \rrbracket-\llbracket e_{1}^{1} \rrbracket \otimes \llbracket e_{1}^{2} \rrbracket\right) .
\end{aligned}
$$

Hence, by Corollary 8.3 , the map $\cdot \nu_{R}: H^{1}(X, R) \rightarrow H^{2}(X, R)$ sends $\llbracket e_{1}^{1} \rrbracket^{*} \mapsto$ $\Phi_{d}(1) \llbracket e_{2} \rrbracket^{*}$ and $\llbracket e_{1}^{2} \rrbracket^{*} \mapsto-\Phi_{d}(1) \llbracket e_{2} \rrbracket^{*}$. On the other hand, the map $\cdot \nu_{R}: H^{0}(X, R)$ $\rightarrow H^{1}(X, R)$ sends $\llbracket e_{0} \rrbracket^{*} \mapsto \llbracket e_{1}^{1} \rrbracket^{*}+\llbracket e_{1}^{2} \rrbracket^{*}$. Recall that we assumed $d$ is not a prime power, i.e., $\Phi_{d}(1)=1$. Therefore, $H^{1}\left(H^{*}(X, R), \nu_{R}\right)=0$.

To recap, we showed that $b_{1}(X, \nu / d)=1$, yet $\beta_{1}\left(X, \nu_{R}\right)=0$. Let us note that $X$ is a minimal CW-complex, in the sense of the definition from 12.1 below; indeed, $\epsilon\left(v_{1}\right)=\epsilon\left(v_{2}\right)=0$, and so the boundary maps in $C \bullet(X, \mathbb{Z})$ vanish. Furthermore, Corollary 6.4 implies that $X$ has the same cohomology ring as $S^{1} \times S^{1}$.

Next, we show that the hypothesis that $H_{*}(X, \mathbb{Z})$ be torsion-free is really necessary.

Example 11.5. Start with $Y=S^{1} \vee S^{2}$ and identify $\pi_{1}(Y)=\mathbb{Z}$, with generator $t$, and set $\Lambda=\mathbb{Z}\left[t^{ \pm 1}\right]$. Using the construction from Example 2.1, build the CW- 
complex $X=Y \cup_{\phi_{1+t}} e_{3}$. The equivariant chain complex of $X$ can be written as

$$
C_{\bullet}(\tilde{X}, \mathbb{Z}): \Lambda \stackrel{1+t}{\longrightarrow} \Lambda \stackrel{0}{\longrightarrow} \Lambda \stackrel{t-1}{\longrightarrow} \Lambda .
$$

Consequently, $C \cdot(X, \mathbb{Z})$ has the form $\mathbb{Z} \stackrel{2}{\rightarrow} \mathbb{Z} \stackrel{0}{\rightarrow} \mathbb{Z} \stackrel{0}{\rightarrow} \mathbb{Z}$, and so $H_{2}(X, \mathbb{Z})=\mathbb{Z}_{2}$.

Now take $\nu: \pi_{1}(X) \rightarrow \mathbb{Z}$ to be the identity, and pick the prime $p=2$. The chain complex $C \bullet\left(X, \mathbb{C}_{\rho}\right)$ corresponding to the rational character $\rho(t)=-1$ has the form $\mathbb{C} \stackrel{0}{\rightarrow} \mathbb{C} \stackrel{0}{\rightarrow} \mathbb{C} \stackrel{-2}{\longrightarrow} \mathbb{C}$, and so $b_{3}(X, \nu / 2)=1$.

On the other hand, it follows from (63) that all boundary maps of $C_{\bullet}\left(X, \mathbb{F}_{2}\right)$ are 0 . Hence, $H_{i}\left(X, \mathbb{F}_{2}\right)=\mathbb{F}_{2}$, generated by $\llbracket e_{i} \rrbracket$, for $0 \leq i \leq 3$. Moreover, $\nu_{\mathbb{F}_{2}}=\llbracket e_{1} \rrbracket^{*}$, the generator of $H^{1}\left(X, \mathbb{F}_{2}\right)=\mathbb{F}_{2}$. We also know from (63) that $\tilde{\partial}_{3}\left(1 \otimes e_{3}\right)=(1+t) \otimes e_{2}$. Hence, the differential $d^{1}: H_{3}\left(X, \mathbb{F}_{2}\right) \rightarrow H_{2}\left(X, \mathbb{F}_{2}\right)$ of $E^{1}\left(X, \mathbb{F}_{2} \mathbb{Z}_{\nu}\right)$ is given by $\llbracket e_{3} \rrbracket \mapsto \llbracket e_{2} \rrbracket$. By Corollary 8.3 , the map $\cdot \nu_{\mathbb{F}_{2}}: H^{2}\left(X, \mathbb{F}_{2}\right) \rightarrow H^{3}\left(X, \mathbb{F}_{2}\right)$ takes $\llbracket e_{2} \rrbracket^{*}$ to $\llbracket e_{3} \rrbracket^{*}$. Hence, $\beta_{3}\left(X, \nu_{\mathbb{F}_{2}}\right)=0$.

11.4. Sharpness of the bound. Inequality (55) from Theorem 11.3 may fail to be an equality, as we now show.

Example 11.6. Let $G=\left\langle x, y \mid[x, y]^{p}\right\rangle$, let $X$ be the associated presentation 2complex, and let $\nu: G \rightarrow \mathbb{Z}$ be the diagonal character, sending both $x$ and $y$ to 1 . We then have $b_{1}(X, \nu / p)=0$, whereas $\beta_{1}\left(X, \nu_{\mathbb{F}_{p}}\right)=1$.

On the other hand, the bound (55) cannot be improved, as the next example shows.

Example 11.7. Let $\mathcal{A}$ be a subarrangement of a complexified reflection arrangement of type $A, B$, or $D$, and let $X$ be the complement of $\mathcal{A}$. Choose $\nu: \pi_{1}(X) \rightarrow \mathbb{Z}$ to be the diagonal character, sending each oriented meridian to 1 . In this case, the bound in Theorem 11.3 is attained at all primes, for $q=1$ and $r=1$; see [19, Theorem $\mathrm{C}]$.

\section{Galois COVERs, minimality, AND LineARIZATiON}

In this section, we analyze in detail the first page of the equivariant spectral sequence of an arbitrary Galois cover. Using this approach, we give an intrinsic meaning to the linearization of the equivariant chain complex, in the important case of minimal $\mathrm{CW}$-complexes. Throughout, $\mathbb{k}$ will denote the integers $\mathbb{Z}$ or a field.

12.1. Minimal cell complexes. We start by reviewing a notion discussed in [23]; see also [7, 8] for various applications. Let $X$ be a connected, finite-type CWcomplex. We say that the CW-structure on $X$ is minimal if the number of $q$-cells of $X$ coincides with the (rational) Betti number $b_{q}(X)$, for every $q \geq 0$. Equivalently, the boundary maps in the cellular chain complex $C_{\bullet}(X, \mathbb{Z})$ are all the zero maps. In particular, $X$ has a single 0 -cell, call it $e_{0}$.

If $X$ is a minimal cell complex, the homology groups $H_{*}(X, \mathbb{Z})$ are all torsionfree. In particular, $H_{q}(X, \mathbb{k})=H_{q}(X, \mathbb{Z}) \otimes \mathbb{k}$ and $H^{q}(X, \mathbb{k})=H_{q}(X, \mathbb{k})^{*}$, for $\mathbb{k}=\mathbb{Z}$ or $\mathbb{k}$ a field.

Even if the homology groups of $X$ are torsion-free, the space $X$ need not be minimal. For example, if $K$ is a knot in $S^{3}$, with complement $X$, then $H_{*}(X, \mathbb{Z})=$ 
$H_{*}\left(S^{1}, \mathbb{Z}\right)$, yet $X$ does not admit a minimal $\mathrm{CW}$-structure unless $K$ is the trivial knot.

Examples of spaces admitting minimal CW-structures are: spheres $S^{n}$, tori $T^{n}$, orientable Riemann surfaces, and complex Grassmanians - in fact, any compact, connected smooth manifold admitting a perfect Morse function. If $\mathcal{A}$ is a complex hyperplane arrangement, then its complement, $X$, has a minimal cell decomposition; see [7].

12.2. Linearizing the equivariant boundary maps. Let $X$ be a minimal $\mathrm{CW}$ complex, with fundamental group $\pi=\pi_{1}(X)$. As usual, let $(C \bullet(X, \mathbb{k}), \partial)$ be the cellular chain complex of $X$, and let $\left(C_{\bullet}(\tilde{X}, \mathbb{k}), \tilde{\partial}\right)$ be the equivariant chain complex, with filtration $F^{n}=I^{n} \cdot C_{\bullet}(\widetilde{X}, \mathbb{k})$, where $I=I_{\mathbb{k}} \pi$. Let $\nu: \pi \rightarrow G$ be an epimorphism, and consider the chain complex $\left(C \bullet\left(X, \mathbb{k} G_{\nu}\right), \tilde{\partial}^{G}\right)$, with filtration $F^{n} C_{\bullet}\left(X, \mathbb{k} G_{\nu}\right)=$ $J^{n} \otimes_{\mathbb{k}} C_{\bullet}(X, \mathbb{k})$, where $J=\bar{\nu}(I)=I_{\mathbb{k}} G$.

Lemma 12.1. $\tilde{\partial}^{G} F^{n} C_{\bullet}\left(X, \mathbb{k} G_{\nu}\right) \subset F^{n+1} C_{\bullet}\left(X, \mathbb{k} G_{\nu}\right)$, for all $n \geq 0$.

Proof. Recall from $₫ 2.4$ that $p \circ \tilde{\partial}=\partial \circ p$, where $p: C \bullet(\tilde{X}, \mathbb{k}) \rightarrow C \bullet(\tilde{X}, \mathbb{k}) / F^{1} C_{\bullet}(X, \mathbb{k})$ is the quotient map. Consequently, $\tilde{\partial} F^{0} \subset F^{1}$, by minimality of $X$.

From the discussion in $\$ 3.4$, we know that $(\nu \otimes$ id $) \circ \tilde{\partial}=\tilde{\partial}^{G} \circ(\nu \otimes$ id $)$ and that $\nu \otimes$ id preserves filtrations. Since $\nu \otimes$ id is onto, we infer that $\tilde{\partial}^{G} F^{0} \subset F^{1}$. The conclusion follows from $\mathbb{k} G$-linearity of $\tilde{\partial}^{G}$.

Definition 12.2. The linearization of the boundary map $\tilde{\partial}^{G}$ is the map induced by $\tilde{\partial}^{G}$ at the associated graded level,

$$
\partial_{G}^{\operatorname{lin}}: \operatorname{gr}_{F}^{*} C_{\bullet}\left(X, \mathbb{k} G_{\nu}\right) \rightarrow \operatorname{gr}_{F}^{*+1} C_{\bullet}\left(X, \mathbb{k} G_{\nu}\right) .
$$

Now again use the minimality of $X$ to identify $C_{q}(X, \mathbb{k})=H_{q}(X, \mathbb{k})$ and

$$
C_{\bullet}\left(X, \mathbb{k} G_{\nu}\right)=\mathbb{k} G \otimes_{\mathbb{k}} H_{q}(X, \mathbb{k}) .
$$

From the construction of the equivariant spectral sequence, we immediately obtain that

$$
E^{1}\left(X, \mathbb{k} G_{\nu}\right)=E^{0}\left(X, \mathbb{k} G_{\nu}\right) \quad \text { and } \quad d_{G}^{1}=\partial_{G}^{\operatorname{lin}} .
$$

At this point, it is easy to give a concrete interpretation of linearization, in terms of matrices and natural $\mathbb{k}$-bases provided by cells. For each $q \geq 1$, denote by $\operatorname{Mat}\left(\tilde{\partial}_{q}^{G}\right)$ the matrix corresponding to the boundary map

$$
\tilde{\partial}_{q}^{G}: \mathbb{k} G \otimes_{\mathbb{k}} H_{q}(X, \mathbb{k}) \rightarrow \mathbb{k} G \otimes_{\mathbb{k}} H_{q-1}(X, \mathbb{k}) .
$$

By Lemma 12.1, all the entries of $\operatorname{Mat}\left(\tilde{\partial}_{q}^{G}\right)$ belong to the ideal $J=I_{\mathbb{k}} G$. Reducing those entries modulo $J^{2}$, we obtain a new matrix, denoted by $\operatorname{Mat}\left(\tilde{\partial}_{q}^{G}\right) \bmod J^{2}$, with entries in $J / J^{2} \cong H_{1}(G, \mathbb{k})$.

Corollary 12.3. Let $X$ be a minimal $C W$-complex, and let $\nu: \pi_{1}(X) \rightarrow G$ be an epimorphism. Then, for all $q \geq 1$,

$$
\operatorname{Mat}\left(\tilde{\partial}_{q}^{G}\right) \bmod J^{2}=\operatorname{Mat}\left(H_{q}(X, \mathbb{k}) \stackrel{\left(\nu_{*} \otimes \mathrm{id}\right) \circ \nabla_{X}}{\longrightarrow} H_{1}(G, \mathbb{k}) \otimes_{\mathbb{k}} H_{q-1}(X, \mathbb{k})\right) .
$$

Proof. With the identifications discussed above, $\operatorname{Mat}\left(\tilde{\partial}_{q}^{G}\right) \bmod J^{2}$ is the matrix of $\partial_{G}^{\operatorname{lin}}=d_{G}^{1}$. The conclusion follows from Proposition 6.3 , 
Remark 12.4. The above equality was proved in [7, Theorem 20] for $\nu=$ id and $\mathbb{k}=\mathbb{Z}$, provided $H^{*}(X, \mathbb{Z})$ is generated as a ring in degree one $\mathbb{2}$ When $\mathbb{k}$ is a field, there is a connection between the chain complex $\left(E^{1}(X, \mathbb{k} \pi), d^{1}\right)$ and the well-known Koszul complex from homological algebra. We refer to [7, Proposition 22] for a class of Koszul resolutions coming from linearization and to [7, Theorem $23]$ for an application to the computation of higher homotopy groups.

12.3. Linearizing the equivariant cochain complex. Let $X$ be a connected CW-complex, with $\pi=\pi_{1}(X)$, and let $\nu: \pi \rightarrow G$ be an epimorphism. Recall from Example 2.3 that

$$
C^{\bullet}\left(X,{ }_{\nu} \mathbb{k} G\right)=\left(\operatorname{Hom}_{\mathbb{k} \pi}\left(C \bullet(\widetilde{X}, \mathbb{k}),{ }_{\nu} \mathbb{k} G\right), \tilde{\delta}_{G}^{\bullet}\right)
$$

denotes the cochain complex of $X$, with coefficients in the left $\mathbb{k} \pi$-module ${ }_{\nu} \mathbb{k} G$. This is a cochain complex of (right) $\mathbb{k} G$-modules, endowed with a decreasing filtration $F^{\bullet}$, with $n$-th term given by

$$
F^{n}=\operatorname{Hom}_{\mathbb{k} \pi}\left(C_{\bullet}(\tilde{X}, \mathbb{k}), J^{n}\right),
$$

with $J=I_{\mathbb{k}} G$ viewed as a left $\mathbb{k} \pi$-module via $\nu$. Alternatively, if we identify $C^{q}\left(X,{ }_{\nu} \mathbb{k} G\right)$ with $\operatorname{Hom}_{\mathbb{k}}\left(C_{q}(X, \mathbb{k}), \mathbb{k} G\right)$, then $F^{n}$ corresponds to $\operatorname{Hom}_{\mathbb{k}}\left(C_{q}(X, \mathbb{k}), J^{n}\right)$.

Lemma 12.5. Suppose $X$ is a minimal $C W$-complex. Then $\tilde{\delta}_{G} F^{n} \subset F^{n+1}$, for all $n \geq 0$.

Proof. Follows immediately from the definition of $\tilde{\delta}_{G}$ and Lemma 12.1 applied to $\nu=\mathrm{id}$.

Thus, the coboundary map $\tilde{\delta}_{G}^{\bullet}$ induces a (dual) linearization map,

$$
\operatorname{gr}_{F}^{*} C^{\bullet}\left(X,{ }_{\nu} \mathbb{k} G\right) \rightarrow \operatorname{gr}_{F}^{*+1} C^{\bullet}\left(X,{ }_{\nu} \mathbb{k} G\right),
$$

or, modulo standard identifications,

$$
\delta_{G}^{\operatorname{lin}}: C^{\bullet}(X, \mathbb{k}) \otimes_{\mathbb{k}} \operatorname{gr}_{J}^{*}(\mathbb{k} G) \longrightarrow C^{\bullet}(X, \mathbb{k}) \otimes_{\mathbb{k}} \operatorname{gr}_{J}^{*+1}(\mathbb{k} G) .
$$

It is a routine matter to verify that the map $\delta_{G}^{\operatorname{lin}}$ above is $\operatorname{gr}_{J}(\mathbb{k} G)$-linear.

Now fix pairs of natural dual bases for $H_{q}(X, \mathbb{k})$ and $H^{q}(X, \mathbb{k})$, for each $q \geq 1$. It is easy to check that $\operatorname{Mat}\left(\tilde{\delta}_{G}^{q}\right)=\operatorname{Mat}\left(\tilde{\partial}_{q}^{G}\right)^{\top}$, where $(\cdot)^{\top}$ denotes the transpose. By Corollary 12.3, then,

$$
\operatorname{Mat}\left(\tilde{\delta}_{G}^{q}\right) \bmod J^{2}=\operatorname{Mat}\left(H_{q}(X, \mathbb{k}) \stackrel{\left(\nu_{*} \otimes \mathrm{id}\right) \circ \nabla_{X}}{\longrightarrow} H_{1}(G, \mathbb{k}) \otimes_{\mathbb{k}} H_{q-1}(X, \mathbb{k})\right)^{\top} .
$$

12.4. Linearization and the Aomoto complex. Finally, we describe the dual linearization map in more familiar terms, in the case when $\nu: \pi \rightarrow \pi_{\mathrm{ab}}$ is the abelianization homomorphism. It turns out that this may be done in terms of the universal Aomoto complex so we begin by reviewing this notion.

Let $H^{*}$ be a graded $\mathbb{k}$-algebra. We need to assume that $H^{1}$ is a free, finitelygenerated $\mathbb{k}$-module, and $a^{2}=0$, for all $a \in H^{1}$. (These conditions are satisfied by the cohomology rings of minimal $\mathrm{CW}$-complexes, with arbitrary coefficients.) Pick a $\mathbb{k}$-basis $\left\{e_{1}^{*}, \ldots, e_{n}^{*}\right\}$ of $H^{1}$, and denote by $\left\{e_{1}, \ldots, e_{n}\right\}$ the dual basis of $H_{1}:=\operatorname{Hom}_{\mathbb{k}}\left(H^{1}, \mathbb{k}\right)$. Denote by $S$ the symmetric algebra $\operatorname{Sym}\left(H_{1}\right)$, and identify it

\footnotetext{
${ }^{2}$ The sign in that theorem comes from considering $C \bullet(X, \mathbb{Z} \pi)$ as a right $\mathbb{Z} \pi$-module instead of a left $\mathbb{Z} \pi$-module; see equations (10) and (13) from [7.
} 
with the polynomial ring $\mathbb{k}\left[e_{1}, \ldots, e_{n}\right]$. The universal Aomoto complex of $H$ is the cochain complex of free $S$-modules,

$$
\mathbb{A}^{\bullet}(H): H^{0} \otimes_{\mathbb{k}} S \stackrel{D^{0}}{\longrightarrow} H^{1} \otimes_{\mathbb{k}} S \stackrel{D^{1}}{\longrightarrow} H^{2} \otimes_{\mathbb{k}} S \stackrel{D^{2}}{\longrightarrow} \cdots,
$$

where the differentials are defined by

$$
D^{q-1}(\alpha \otimes 1)=\sum_{i=1}^{n} e_{i}^{*} \cdot \alpha \otimes e_{i}
$$

for $\alpha \in H^{q-1}$ and then extended by $S$-linearity. Our hypothesis on (strong) anticommutativity of $H^{*}$ in degree one easily implies that $D \circ D=0$.

The terminology is motivated by the following universal property of $\mathbb{A}^{\bullet}$. Pick any element $z \in H^{1}=\operatorname{Hom}_{\mathbb{k}}\left(H_{1}, \mathbb{k}\right)$ and denote by $\mathrm{ev}_{z}: S \rightarrow \mathbb{k}$ the change of rings given by evaluation at $z$. This leads to a specialization of $\mathbb{A}^{\bullet}$, namely to the $\mathbb{k}$-cochain complex $\mathbb{A}^{\bullet}(z):=\mathbb{A}^{\bullet} \otimes_{S} \mathbb{k}$. It is easy to check that $\mathbb{A}^{\bullet}(z)$ coincides with the Aomoto complex of $H$ with respect to $z$, as defined in 9.2 We are now in a position to state the last result of this paper.

Theorem 12.6. Let $X$ be a minimal $C W$-complex. Then the linearization of the equivariant cochain complex of the universal abelian cover of $X$, with coefficients in $\mathbb{k}=\mathbb{Z}$ or a field, coincides with the universal Aomoto complex of the cohomology $\operatorname{ring} H^{*}(X, \mathbb{k})$.

Proof. The dual linearized complex is described by (64) and (65). More precisely, for each $q \geq 1$, the transposed matrix of

$$
\delta_{\pi_{\mathrm{ab}}}^{\operatorname{lin}}: H^{q-1}(X, \mathbb{k}) \otimes_{\mathbb{k}} S \rightarrow H^{q}(X, \mathbb{k}) \otimes_{\mathbb{k}} S
$$

coincides with the matrix of

$$
\nabla_{X}: H_{q}(X, \mathbb{k}) \rightarrow H_{1}(X, \mathbb{k}) \otimes_{\mathbb{k}} H_{q-1}(X, \mathbb{k}) .
$$

On the other hand, $\nabla_{X}$ is the transpose of $\bigcup_{X}$. Thus, $\delta_{\pi_{\mathrm{ab}}}^{\operatorname{lin}}$ coincides with the differential $D^{q-1}$ from (66).

\section{ACKNOWLEDGMENTS}

This work was started while the first author visited Northeastern University, in Spring, 2006. He thanks the Northeastern Mathematics Department for its support and hospitality during this visit. A substantial portion of the work was done while both authors visited the Abdus Salam International Centre for Theoretical Physics in Trieste, Italy, in Fall, 2006. We thank the ICTP for its support and excellent facilities.

\section{REFERENCES}

[1] J. Birman, Braids, links and mapping class groups, Ann. of Math. Studies, vol. 82, Princeton Univ. Press, Princeton, NJ, 1975. MR0375281

[2] H. Cartan, S. Eilenberg, Homological algebra, Princeton University Press, Princeton, NJ, 1956. MR0077480

[3] D. C. Cohen, On the cohomology of discriminantal arrangements and Orlik-Solomon algebras, in: Arrangements-Tokyo 1998, pp. 27-49, Adv. Stud. Pure Math., vol. 27, Kinokuniya, Tokyo, 2000. MR:1796892 (2001m:32059)

[4] D. C. Cohen, P. Orlik, Arrangements and local systems, Math. Res. Lett. 7 (2000), no. 2-3, 299-316. MR1764324(2001i:57040) 
[5] D. C. Cohen, A. I. Suciu, Characteristic varieties of arrangements, Math. Proc. Cambridge Phil. Soc. 127 (1999), no. 1, 33-53. MR.1692519 (2000m:32036)

[6] G. Denham, The Orlik-Solomon complex and Milnor fibre homology, Topology Appl. 118 (2002), no. 1-2, 45-63. MR.1877715 (2002k:32048)

[7] A. Dimca, S. Papadima, Hypersurface complements, Milnor fibers and higher homotopy groups of arrangements, Annals of Math. 158 (2003), no. 2, 473-507. MR2018927 (2005a:32028)

[8] A. Dimca, S. Papadima, Equivariant chain complexes, twisted homology and relative minimality of arrangements, Ann. Sci. École Norm. Sup. 37 (2004), no. 3, 449-467. MR2060483 (2005c:55028)

[9] A. Dimca, S. Papadima, A. Suciu, Alexander polynomials: Essential variables and multiplicities, Int. Math. Res. Notices 2008, no. 3, Art. ID rnm119, 36 pp. MR2416998(2009i:32036)

[10] S. Eilenberg, Homology of spaces with operators. I. Trans. Amer. Math. Soc. 61 (1947), 378-417. MR0021313

[11] M. S. Farber, Sharpness of the Novikov inequalities, Funktsional. Anal. i Prilozhen. 19 (1985), no. 1, 49-59. MR.0783706 (86g:58029)

[12] M. Farber, Topology of closed one-forms, Math. Surveys Monogr., vol. 108, Amer. Math. Soc., Providence, RI, 2004. MR2034601 (2005c:58023)

[13] R. H. Fox, Free differential calculus. I. Derivation in the free group ring, Ann. of Math. 57 (1953), 547-560. MR0053938

[14] L. Grünenfelder, Lower central series, augmentation quotients and homology of groups, Comment. Math. Helv. 55 (1980), no. 2, 159-177. MR0576599 (82a:20044)

[15] G. Higman, The units of group-rings, Proc. London Math. Soc. (2) 46 (1940), 231-248. MR0002137

[16] J. Hillman, A link with Alexander module free which is not a homology boundary link, J. Pure Appl. Algebra 20 (1981), no. 1, 1-5. MR0596149(82d:57001)

[17] P. Hilton, U. Stammbach, A course in homological algebra, Second edition, Graduate Texts in Math., vol. 4, Springer-Verlag, New York, 1997. MR1438546 (97k:18001)

[18] J. Howie, H. Short, The band-sum problem, J. London Math. Soc. (2) 31 (1985), no. 3, 571-576. MR0812788 (87c:57004)

[19] A. Măcinic, S. Papadima, On the monodromy action on Milnor fibers of graphic arrangements, Topology Appl. 156 (2009), no. 4, 761-774. MR2492960

[20] H. Matsumura, Commutative algebra, Second edition, Math. Lecture Note Ser., vol. 56, Benjamin/Cummings, Reading, MA, 1980. MR0575344 (82i:13003)

[21] J. Milnor, Infinite cyclic coverings, in: Conference on the Topology of Manifolds (Michigan State Univ., 1967), pp. 115-133, Prindle, Weber \& Schmidt, Boston, MA, 1968. MR0242163 $(39: 3497)$

[22] S. P. Novikov, Bloch homology, critical points of functions, and closed 1-forms, Dokl. Akad. Nauk. SSSR 287 (1986), 1321-1324. MR.0838822 (87k:58052)

[23] S. Papadima, A. I. Suciu, Higher homotopy groups of complements of hyperplane arrangements, Advances in Math. 165 (2002), no. 1, 71-100. MR1880322 (2003b:55019)

[24] S. Papadima, A. I. Suciu, Toric complexes and Artin kernels, Advances in Math. 220 (2009), no. 2, 441-477. MR2466422

[25] S. Papadima, A. I. Suciu, Algebraic monodromy and obstructions to formality, arxiv:0901.0105, to appear in Forum Math (2010).

[26] D. Quillen, On the associated graded ring of a group ring, J. Algebra 10 (1968), 411-418. MR0231919

[27] A. Reznikov, Three-manifolds class field theory (homology of coverings for a nonvirtually $b_{1}$ positive manifold), Selecta Math. (N.S.) 3 (1997), no. 3, 361-399. MR.1481134 (99b:57041)

[28] D. Rolfsen, Knots and links, Math. Lecture Series, vol. 7, Publish or Perish, 1976. MR0515288

[29] H. Seifert, Über das Geschlecht von Knoten, Math. Ann. 110 (1935), no. 1, 571-592. MR.1512955

[30] J.-P. Serre, Algèbre locale. Multiplicités, Third edition, Lecture Notes in Math., vol. 11, Springer-Verlag, Berlin, 1975. MR0201468

[31] E. H. Spanier, Algebraic topology, McGraw-Hill, New York-Toronto-London, 1966. MR0210112 
[32] J. R. Stallings, Quotients of the powers of the augmentation ideal in a group ring, in: Knots, groups, and 3-manifolds, pp. 101-118, Ann. of Math. Studies, no. 84, Princeton Univ. Press, Princeton, N.J., 1975. MR0379685(52:590)

[33] S. Wanna, A spectral sequence for group presentations with applications to links, Trans. Amer. Math. Soc. 261 (1980), no. 1, 271-285. MR0576875 (81i:57003)

[34] J. H. C. Whitehead, Combinatorial homotopy (I and II), Bull. Amer. Math. Soc. 55 (1949), 213-245, 453-496. MR0030759(11:48b) MR0030760(11:48c)

[35] M. Yoshinaga, The chamber basis of the Orlik-Solomon algebra and Aomoto complex, Arkiv för Matematik, 47 (2009), no. 2, 393-407. MR2529708

Institute of Mathematics Simion Stollow, P.O. Box 1-764, RO-014700 Bucharest, RoMANIA

E-mail address: Stefan.Papadima@imar.ro

Department of Mathematics, Northeastern University, Boston, Massachusetts 02115

E-mail address: a.suciu@neu.edu

URL: http://www.math.neu.edu/ suciu 